\title{
The Distribution of Internal Interfaces in Polycrystals
}

\author{
Brent L. Adams \\ b_I_adams@byu.edu \\ Bassem S. El-Dasher \\ Gregory S. Rohrer \\ Anthony D. Rollett \\ David M. Saylor
}

See next page for additional authors

Follow this and additional works at: https://scholarsarchive.byu.edu/facpub

Part of the Mechanical Engineering Commons

Original Publication Citation

Zeitschrift fur Metallkunde, 95 (24) 197-214

\section{BYU ScholarsArchive Citation}

Adams, Brent L.; El-Dasher, Bassem S.; Rohrer, Gregory S.; Rollett, Anthony D.; Saylor, David M.; and Wynblatt, Paul, "The Distribution of Internal Interfaces in Polycrystals" (2003). Faculty Publications. 467. https://scholarsarchive.byu.edu/facpub/467 accepted for inclusion in Faculty Publications by an authorized administrator of BYU ScholarsArchive. For more information, please contact ellen_amatangelo@byu.edu. 


\section{Authors}

Brent L. Adams, Bassem S. El-Dasher, Gregory S. Rohrer, Anthony D. Rollett, David M. Saylor, and Paul Wynblatt 


\title{
The Distribution of Internal Interfaces in Polycrystals
}

Gregory S. Rohrer, David M. Saylor ${ }^{\mathrm{a}}$, Bassem El Dasher ${ }^{\mathrm{b}}$, Brent L. Adams ${ }^{\mathrm{c}}$, Anthony D. Rollett, and Paul Wynblatt.

Department of Materials Science and Engineering, Carnegie Mellon University, Pittsburgh, Pennsylvania 15213-3890

${ }^{a}$ National Institute of Standards and Technology, Gaithersburg, MD 20899, USA

bcurrent address: University of California, Lawrence Livermore National Laboratory, P.O. Box 808, Livermore, CA 94511

${ }^{\mathrm{c}}$ Department Mechanical Engineering, Brigham Young University, Provo, UT 84602, USA

\begin{abstract}
Recent advances both in experimental instrumentation and computing power have made it possible to interrogate the distribution of internal interfaces in polycrystals and the three dimensional structure of the grain boundary network with an unprecedented level of detail. The purpose of this paper is to review techniques that can be used to study the mesoscopic crystallographic structure of grain boundary networks and to summarize current findings. Recent studies have shown that grain surfaces within dense polycrystals favor the same low energy planes that are found on equilibrium crystal shapes and growth forms of crystals in contact with another phase. In the materials for which comprehensive data exists, the distribution of grain boundaries is inversely correlated to the sum of the energies of the surfaces of the grains on either side of the boundary.
\end{abstract}

Keywords, Grain boundaries, Surface energy, Grain boundary energy, Polycrystals Version 11/30/03, submitted to Zeitschrift für Metallkunde 


\section{Introduction}

Dense polycrystalline materials consist of irregularly shaped, approximately polygonal, single crystals joined at internal interfaces referred to as grain boundaries. The internal structure of a polycrystal is most frequently characterized by observing planar sections in which the grain boundary planes appear as lines. Therefore, knowledge of the three-dimensional shapes of grains and the connectivity of the interfacial network is limited. During the last decade, however, there have been significant advances both in experimental instrumentation and computing power. Because it is now possible to collect and analyze large quantities of observations in an automated fashion and to model structures of increasing complexity, studies of the threedimensional structure of interfacial networks have begun to flourish. The purpose of this paper is to review what is known about the structure of internal interfaces in polycrystals and to put recent results in the context of more historical studies. The scope will be confined to the macroscopic and crystallographic structure of interfaces, and will concentrate on single phase materials. In the next section, the observable macroscopic characteristics of grain boundaries in polycrystals are defined. Methods used to study the structure of three dimensional interfacial networks are then outlined in Section 3, and this is followed by a summary of recent findings in Section 4. In the final section, the most important points are reviewed and several interesting directions for future research are identified. 


\section{Observable Characteristics of the Interfacial Network}

\subsection{Grain boundary degrees of freedom}

To distinguish one type of grain boundary from another, the values of five independent parameters must be specified [1]. Throughout the majority of this review, three parameters will be used to describe the lattice misorientation $(\square g)$ across the boundary and two parameters will be used to describe the interface normal (n). The parameters needed to specify the distribution of internal grain surfaces are described are described in Section 2.2. This distribution is a function of the two parameters associated with n. In section 2.3, the distribution of internal grain surfaces will be considered as a function of the lattice misorientation. This is a five parameter function that is referred to as the grain boundary character distribution.

\subsection{Distribution of internal grain surfaces, $\square(\boldsymbol{n})$}

To define the internal distribution of internal grain surfaces, we can begin by considering a volume of material with a constant orientation, enclosed by curved surfaces that meet along lines where there is an abrupt change in the surface normal, as illustrated in Fig. 1a [2]. If the curvature of the surfaces between grains is approximated by a set of triangular tangent planes with a fixed surface orientation, then a grain can be defined as a polyhedral volume of material with constant orientation. Referring to Fig. $1 \mathrm{~b}$ and $\mathrm{c}$, the vertices of these triangular tangent planes have coordinates $\left(\mathrm{x}_{\mathrm{k}}, \mathrm{y}_{\mathrm{k}}, \mathrm{z}_{\mathrm{k}}\right)$. These coordinates are measured in the sample reference frame, which is relative to the external surfaces of the polycrystal or, more generally, the microscope stage. For the $\mathrm{j}^{\text {th }}$ facet on the $\mathrm{i}^{\text {th }}$ grain, 
we can define the vectors $\mathbf{r}_{\mathrm{ij} 1}$, directed from $\mathrm{x}_{1}, \mathrm{y}_{1}, \mathrm{z}_{1}$ to $\mathrm{x}_{2}, \mathrm{y}_{2}, \mathrm{z}_{2}$ and $\mathbf{r}_{\mathrm{ij} 2}$ directed from $\mathrm{x}_{1}, \mathrm{y}_{1}, \mathrm{z}_{1}$ to $\mathrm{x}_{3}, \mathrm{y}_{3}, \mathrm{z}_{3}$. Therefore, the normal to this tangent plane is:

$$
\mathbf{n}_{\mathrm{ij}}^{\prime}=\frac{\mathbf{r}_{\mathrm{ij} 1} \square \mathbf{r}_{\mathrm{ij} 2}}{\left|\mathbf{r}_{\mathrm{ij} 1} \square \mathbf{r}_{\mathrm{ij} 2}\right|}
$$

where $\mathbf{n}^{\prime}{ }_{\mathrm{ij}}$ is a unit vector in the sample reference frame.

To specify crystallographic orientations, we use three Eulerian angles, $\square_{1}, \square$, and $\square$. These angles describe a series of three rotations that transform the sample reference axes into alignment with the crystal axes. This transformation is denoted by $g\left(\square_{1}, \square, \square_{2}\right)$, a $3 \times 3$ matrix that can be used to convert vectors observed in the sample reference frame to the crystal reference frame, $\mathbf{n}_{\mathrm{ij}}=\boldsymbol{g} \mathbf{n}_{\mathrm{ij}}$ [3]. In this process, it is important to recognize crystal symmetries. If there are $\mathrm{M}$ symmetry operators in the crystal system, then for each observed $\mathbf{n}^{\prime}{ }_{\mathrm{ij}}$ there are $\mathrm{M}$ indistinguishable vectors in the crystal reference frame that are given by $\mathbf{n}_{\mathrm{ij}}=\mathrm{C}_{\mathrm{m}} g \mathbf{n}_{\mathrm{ij}}$, where the $\mathrm{C}_{\mathrm{m}}$ are the $\mathrm{M}$ symmetry operators. We can then define $\square(\mathbf{n})$, the distribution of internal grain surfaces, as the area of all internal surfaces with orientation $\mathbf{n}$, normalized by the average area per orientation. If the distribution were random, $\square$ would be constant for all values of $\mathbf{n}$. Variations in $\square$ indicate a preference for certain grain surfaces.

To measure $\square(\mathbf{n})$, it is convenient to partition the domain of possible grain surface normals into discrete cells. For centrosymmetric crystals, two spherical angles ( $\square$ and $\square$ ) in the range between 0 and $\square / 2$ and 0 and $2 \square$, respectively, reproduce the domain of possible grain surface normals. So that each cell has the same area on the surface of a 
unit sphere, the domain of $\mathbf{n}$ is parameterized by $\cos \square$ and $\square$, as illustrated in Fig. 2. If there are D cells for every $\square / 2$ radians, then there will be a total of D•4D cells, each with the size $\square \cos \square=1 / \mathrm{D}$ and $\square \square=2 \square / 4 \mathrm{D}$. This equal area parameterization is useful because every cell has the same probability of being populated by a random internal grain surface orientation, $\mathbf{n}$. As the internal surfaces in a polycrystal are characterized, the area of each observed triangle is added to cells in the domain of $\square(\mathbf{n}) \mathrm{M} / 2$ times (the factor of 2 arises because the crystal is assumed to have a center of symmetry and only the upper hemisphere of surface orientation space is considered). At the end, the area in each cell can be divided by the average area per cell to produce results in units of multiples of a random distribution (MRD). If the distribution of internal grain surfaces were random, then the value in each cell would be unity. Values greater than unity occur when the total area associated with a specific type of internal surface is larger than would be expected in a random distribution; values less than unity are associated with internal surfaces whose total areas are less than expected in a random distribution. As an example, a plot of $\square(\mathbf{n})$ for $\mathrm{MgO}$ is illustrated in Fig. 3 [4]. In this case, we see that internal surfaces with the $\{100\}$ orientation are observed more than twice as frequently as expected in a random distribution and those with $\{111\}$ orientation are observed less frequently.

\subsection{The grain boundary character distribution, $\square(\square g, \boldsymbol{n})$}

Because each triangular segment is the interface between two misoriented grains, it will be considered twice in the measurement of $\square(\mathbf{n})$ : once when associated with the crystal orientation on one side of the interface $\left(g_{1}\right)$ and again when associated with the crystal orientation on the other side $\left(g_{2}\right)$. In other words, the distribution of internal grain 
surfaces is measured independently of the boundary misorientation. Since we know the crystal orientations on either side of the boundary, we can calculate the misorientation, $\square g=\mathrm{g}_{1} \mathrm{~g}_{2}{ }^{\mathrm{T}}$, and measure the quantity we refer to as the grain boundary character distribution function, $\square(\square g, \mathbf{n})$. While the distribution of internal grain surfaces, $\square(\mathbf{n})$, has the same symmetry as the crystal, $\square(\square g, \mathbf{n})$ depends on five independent parameters and the symmetry is more complex. Here we apply the bicrystal symmetry originally defined by Morawiec [5]. The misorientation must be calculated with respect to both grains:

$$
\begin{aligned}
& \square g=C_{p 1} g_{1}\left(C_{p 2} g_{2}\right)^{T} \quad \text { and, } \\
& \square g=C_{p 2} g_{2}\left(C_{p 1} g_{1}\right)^{T}
\end{aligned}
$$

For crystals with a center of symmetry, this leads to $2 \mathrm{P}^{2}$ equivalent misorientations, where $\mathrm{P}=\mathrm{M} / 2$ is the number of proper symmetry operators. For each indistiguishable $\square g$, we calculate the plane normal in the crystal reference frame $\left(\mathbf{n}_{\mathrm{ij}}\right)$ using the measured planar normal in the sample frame $\left(\mathbf{n}_{\mathrm{ij}}{ }^{\prime}\right)$ and the non-transposed $g_{\mathrm{i}}$. In other words, when $\square g$ is calculated using Eq. $2 \mathrm{a}, \mathbf{n}_{\mathrm{ij}}=\mathrm{C}_{\mathrm{p} 1} g_{1} \mathbf{n}_{\mathrm{ij}}{ }^{\prime}$, and when $\square g$ is calculated using Eq. $2 \mathrm{~b}, \mathbf{n}_{\mathrm{ij}}=$ $\mathrm{C}_{\mathrm{p} 2} g_{2} \mathbf{n}_{\mathrm{ij}}$. Finally, we note that it is arbitrary whether the grain boundary normal points into the first or second crystal. This adds an additional factor of two to the number of symmetrically equivalent boundaries so that $2 \cdot 2 \cdot \mathrm{P}^{2}$ symmetrically equivalent grain boundaries are generated from a single triangular interface segment.

As with $\square(\mathbf{n})$, it is convenient to represent $\square(\square g, \mathbf{n})$ as a finite set of discrete grain boundaries types. The three Eulerian angles $\left(\square_{1}, \square, \square_{2}\right)$ used to specify $\square g$ can be parameterized by $\square_{1}, \cos (\square)$, and $\square_{2} ; \mathbf{n}$ can be parameterized using the same spherical 
angle convention used for $\square(\mathbf{n})$ (see Section 2.2). The choice of the size of the domain depends on the crystal symmetry. In the complete domain, the five angular parameters, $\square_{1}, \square, \square_{2}, \square$, and $\square$ range from 0 to $2 \square, \square, 2 \square, \square$, and $2 \square$, respectively. For materials with cubic or tetragonal symmetry, it is sufficient to use a sub-domain in which the misorientation parameters range from zero to $\square / 2,1$, and $\square / 2$ for $\square_{1}, \cos (\square)$, and $\square_{2}$ respectively. This sub-domain is $1 / 64$ th of the entire range of possibilities and is a convenient choice because it is the smallest volume that contains an integer number of fundamental zones and can still be partitioned in a simple way. For the cubic system, there are $2304\left(=2 \cdot 2 \cdot 24^{2}\right)$ general equivalent grain boundaries for every observed triangular segment and $36(=2304 / 64)$ of these are in the sub-domain. So, each observation is represented by 36 indistinguishable symmetrically equivalent boundaries. In the tetragonal system, there are $256\left(=2 \cdot 2 \bullet 8^{2}\right)$ general equivalent grain boundaries for every observed triangular segment and $4(=256 / 64)$ of these are in the sub-domain. The choice of parameters allows the sub-domain to be partitioned into cells of equal volume when the parameters are equally partitioned. In this case, there are $\mathrm{D}^{3}$ cells with $\overline{\mathrm{C}} \mathrm{D}_{\mathrm{l}}=$ $\square \square_{2}=\square / 2 \mathrm{D}$ and $\square \cos \square=1 / \mathrm{D}$. The cells in Euler space can be visualized as a three dimensional rectangular parallelepiped, as illustrated in Fig. 2b. Using the previously described discretization for $\mathbf{n}$, there are also $4 \mathrm{D}^{2}$ boundary plane normals for each cell in the misorientation domain.

\subsection{Number of distinguishable grain boundary types}

Because our discrete representation of $\square(\square g, \mathbf{n})$ is five-dimensional, the number of different boundary types increases dramatically as the cell size is decreased. Here we 
estimate how the number of distinguishable boundary types varies with the resolution of the five angular parameters. Although the parameterization of the complete domain described above is nonuniform and leads to singularities, we can estimate the number of different boundaries in the following way. If each of these parameters can be resolved with accuracy, $\square$, then the number of different boundaries is found by dividing the total volume $\left(8 \square^{5}\right)$ by the volume of one resolved boundary, $\square^{5}$. Crystal symmetry makes many of these boundaries will be indistinguishable, so the total must be divided by the number of symmetrically equivalent bicrystals. Therefore, the total number of distinguishable boundaries will be:

$$
N=\frac{8 \square^{5}}{4 \mathrm{P}^{2} \square^{5}}
$$

This expression is approximate, because the spacing of the cells is not commensurate with the spacing of the fundamental zones within the sub-domain. This creates some degeneracy in the cells crossing the edges where multiple fundamental zones meet. To correct for this effect, a term should be subtracted from Eq. 3 that scales approximately with $1 / \square^{3}$. Since $\square<1,1 / \square^{5}>>1 / \square^{3}$, and the effect on $N$ is not significant as long as $\square$ is small. The number of distinguishable grain boundaries given by Eq. 3 is plotted as a function of $\square$ in Fig. 4 for cubic, tetragonal, and hexagonal crystals.

Since the number of distinguishable grain boundaries is strongly sensitive to the resolution, the relevant range for $\square$ should be considered. Based on studies of grain boundary properties, such as the excess free energy per area, there are significant variations over angular ranges as small as $10^{\circ}[6]$. On the other hand, it is currently not 
routine to measure of $\mathbf{n}$ with an accuracy better than $5^{\circ}$. Therefore, we take the range of $\square$ between $5^{\circ}$ and $10^{\circ}$ as the relevant range. The graph in Fig. 4 shows that the number of distinguishable boundary types is very large in this range. For example, at a resolution of $10^{\circ}$, there are approximately $6.5 \times 10^{3}$ distinct boundaries in the cubic system. If the resolution is increased to $5^{\circ}$, there are $2 \times 10^{5}$ distinguishable boundaries. The important point is that if $\square(\square g, \mathbf{n})$ is to be reliably measured, the number of observed grain boundaries must be larger than $\mathrm{N}$.

\subsection{Representation of the grain boundary character distribution}

There are a number of potential ways of parameterizing and displaying the fivedimensional grain boundary distribution. By separating the three misorientation parameters $(\square \mathrm{g})$ and the two interface plane parameters $(\mathbf{n})$, the distribution of grain boundary planes at each misorientation can be plotted on a stereographic projection, which is a familiar crystallographic tool. Among the potential ways of representing the misorientation, which have been described by Frank [7], the axis-angle convention is probably the most familiar. All bicrystals have a common axis that has the same direction in the reference frame of both crystals. Rotations about this axis, [uvw], take the two crystals from the coincident configuration at $\square=0$ to misoriented configurations of $\square$ about [uvw]. So, for any axis-angle combination, a distribution of grain boundary planes can be plotted as illustrated in Fig. 5, which shows the distribution of grain boundary planes in $\mathrm{MgO}$ for boundaries misoriented by $45^{\circ}$ around [110]. The data in (a) are plotted in projection along the [001] direction [4]. In this case, the misorientation axis lies in the plane; pure twist boundaries are those with planes parallel to the common 
axis and pure tilt boundaries are those with planes perpendicular to the common axis. Both are marked on the figure. Using this method, each point in the misorientation space must be examined individually.

Each method for parameterizing the five-dimensional space of grain boundary types has certain strengths and weaknesses. For example, a weakness of the Euler angle parameterization is that at small misorientation angles, all of the axes converge towards a point and are no longer discriminated. If the resolution is $10^{\circ}$, then all interfaces with $=5^{\circ}$ will come from the same set of equivalent cells in Euler space, regardless of [uvw]. Alternate schemes can eliminate this particular problem, but creates other difficulties. Therefore, it is important to select the parameterization scheme that is most suited to the question at hand. One alternate representation that has found application is the so-called interface plane scheme, where each grain boundary is characterized by the vectors normal to the surfaces of the two grains on either side of the boundary $\left(\mathbf{n}_{1}\right.$ and $\left.\mathbf{n}_{2}\right)$, and a twist angle, $\square[8]$. Referring back to Fig. 1, this is a natural way to characterize the interface, since the grain boundary can always be thought of as two joined surfaces.

Experimental observation of $\square\left(\mathbf{n}_{1}, \mathbf{n}_{2}, \square\right)$ and visualization are again aided by discretization of the five dimensional space. In the cubic system, all vectors $\mathbf{n}_{\mathbf{1}}$ can be represented in a single standard stereographic triangle. Takashima et al. [9] selected 12 discrete poles in the $(100)-(110)-(111)$ triangle (see Fig. 6a). Two triangles are required for the representation of $\mathbf{n}_{2}$, so the (100) - (101) - (111) triangle and two additional poles were added, as illustrated in Fig. 6b. To examine $\square\left(\mathbf{n}_{1}, \mathbf{n}_{2}, \square\right)$, a pole is selected for $\mathbf{n}_{\mathbf{1}}$ and $\mathbf{n}_{\mathbf{2}}$ and $\square$ is plotted as a function of $\square$ in the range from 0 to $2 \square$. Using this discretization, there are 81 distinct combinations of $\mathbf{n}_{\mathbf{1}}$ and $\mathbf{n}_{\mathbf{2}}$. Figure $6 \mathrm{~b}$ shows the 
frequency of observed (111)\|(111) twist boundaries in a Fe-Mn-Cu alloy [10]. The minimum misorientation is also plotted to illustrate that the population peaks as the positions of the coherent twin (in the axis-angle space, this is $60^{\circ}$ around [111]) and for low angle grain boundaries.

\subsection{Bicrystal symmetries}

The data in Figs. 5 and 6 suggests that the distribution of grain boundary planes at a fixed misorientation can have some symmetry and that it is not always necessary to consider the entire range of parameters. However, this happens only in special cases where axes with common symmetry elements from each of the two crystals are parallel. For the case of a general bicrystal, the unique zone of boundary planes for a general fixed misorientation is a hemisphere $(0 \leq \square<\square / 2,0<\square<2 \square)$ [5]. The special cases occur when rotational symmetry elements in the two crystals are parallel; in these cases, the bicrystal has additional symmetry that results from combining the common rotational symmetry with operations that relate positions in the two crystals without regard for the crystal of origin [11,12]. If $n$-fold rotational axes of symmetry in both crystals are parallel, and the crystal is centrosymmetric, then the range of unique planes is reduced such that $0 \leq \square \leq$ $\square / \mathrm{n}$, where $\square$ is defined as the azimuthal angle about the axis of symmetry originating at a symmetric tilt boundary (the polar angle $\square$, away from the direction of the symmetry axis, remains in the range of $0 \leq \square \leq \square / 2$ ).

This extra symmetry is apparent in Fig. 5, which shows the grain boundary plane distribution for bicrystals with a $45^{\circ}$ rotation about [110]. Here the crystals share the [110] axis which has $2 / \mathrm{m}$ symmetry. The fact that exchanging the crystals on either side 
of the boundary produces an indistinguishable bicrystal leads to extra symmetry and, in this case, all bicrystals with a common [110] axis have a point symmetry of at least mmm. For example, at the $45^{\circ}$ rotation about [110] shown in Fig. 5, there are two mirrors crossing in the plane of projection that result from the interchange of the crystals and this makes the point symmetry mmm. The symmetry is not always obvious in the [001] projection, so Fig. 5b shows the same data projected alone the [110] axis. It should be noted that while the numerical data has the ideal mmm symmetry, the contouring routine used to plot the data introduces some small deviations from ideality that can be detected in careful inspections of the stereograms in this paper. The $\overline{3}$ symmetry of the [111] axis means that bicrystals misorientated about this direction have at least $\overline{3} \mathrm{~m}$ symmetry; for $60^{\circ}$ rotations about this axis, the symmetry is $6 / \mathrm{m}$. It is interesting to note that at some special points, the bicrystals can have noncrystallographic point symmetries. For example, all bicrystals in the cubic system with [100] misorientation axes have at least $4 / \mathrm{mmm}$ point symmetry, but for the $45^{\circ}$ rotation, $\square(\mathbf{n})$ has a point symmetry of $8 / \mathrm{m}$, as illustrated in Fig. 7. It should be emphasized that these special symmetries arise only for misorientations where compatible rotational symmetry axes from both crystals are parallel. For the cubic case, this occurs only for $\langle 100\rangle$, $\langle 110\rangle$, and $\langle 111\rangle$ misorientations and when $<100>\| 1<110>$. 


\section{Experimental Methods for Measuring the Distributions in Internal Interfaces in Polycrystals}

\subsection{Direct Observation of Grain Shape}

The direct, optical observation of three-dimensional grain shapes in polycrystals is usually not possible. If the material is transparent, and a section smaller than the grain size is viewed in transmission, then it is possible to visualize the inclination of the grain boundaries. However, for thicker and opaque samples, light absorption and scattering become limiting factors. To get around the problem of direct observation, foams (soap films) have long been studied as analogs to polycrystals [13-16]. The property that a soap film network has in common with the grain boundary network is that the interfaces adopt configurations driven by the balance of surface tensions. For the case of foams, the film boundaries must all meet at triple lines with $120^{\circ}$ dihedral angles and the triple lines must all meet at quadrajunctions with $109.5^{\circ}$ dihedral angles; these configurations balance the surface tensions and are in local mechanical equilibrium. Since there is no regular polyhedron with planar sides that simultaneously meets these criterion and fills space, the bubbles must change shape and have curved boundaries supported by pressure differences. One other interesting polycrystal analog is lead shot, which has been compressed to a minimum volume to form polyhedral bodies that can then be separated and analyzed [17].

While bubbles in foams and grains in polycrystals are topologically analogous, there are two critical differences. The first is that the capillary pressure that drives grain boundary motion is not analogous to the gas pressure within a bubble [18]. Rapid motion 
of gas within a bubble makes it impossible to sustain internal pressure differences and the uniform pressure will lead to a constant mean curvature on the boundaries of the bubble. Rapid transport of the crystalline solid is not possible and, as a result, the capillary pressure is not expected to be uniform and can be determined by local conditions. Therefore, the shapes of crystalline grains are not expected to be bounded by surfaces with a uniform curvature. The second important difference is that while the interface energy per unit area of the soap film is isotropic, this is not so in the crystal. In general, the energy per unit grain boundary area $(\square$ in the polycrystal is a function of the five macroscopic parameters: $\square \square g, \mathbf{n})$. The condition for local equilibrium at the interface are now given by the Herring equation [19]:

$$
\square_{i} \square_{i} \hat{t}_{i}+\frac{\partial \square_{i}}{\partial \square_{i}} \hat{n}_{i}=0
$$

where $\square$ is the right-handed angle of rotation about the triple line direction, measured from a reference direction and the other terms are defined in Fig. 8. This equilibrium condition permits a wide range of dihedral angles that are expected to deviate significantly from $120^{\circ}$. Furthermore, grain surfaces will adjust to increase the area of low energy boundary orientations while decreasing the energy of higher energy boundary orientations. For these reasons, it is necessary to study the interfaces of actual crystalline grains, rather than soap bubbles.

In several systems, intact grains can be separated from the polycrystal and examined individually. When a $\square$-brass casting is placed in a warm solution of dilute nitric acid, and mercurous nitrate is added, a film of mercury forms on the surfaces and 
this allows the grains to be separated [20-22]. It is also possible to separate the grains in a fine -brass casting by crushing at about $400{ }^{\circ} \mathrm{C}$, when it is brittle [23]. The grains of a stainless steel have been separated by selective grain boundary corrosion [24] and the grains in aluminum can be separated from the polycrystal by infiltration of liquid gallium [25]. The most complete study of separated $\mathrm{Q}$-brass grains was completed by Hull [22], who examined 941 separated whole grains. So far, these direct observations of separated grains have provided information only on the shapes of grains, not the crystallographic orientations of the internal surfaces.

\subsection{Optical Measurements in Thin Section}

As mentioned earlier, light microscopy can be used to measure grain shapes in thin samples that transmit light. By viewing a crystal in transmitted light through crossed polarizers with a lambda plate inserted, regions of constant orientation in the microstructure appear with constant interference colors so that individual grains in the microstructure can be distinguished on the basis of their color. The color of a grain depends on the thickness of the sample, the material's birefringence, and the orientation of the optical axis. Unfortunately, there is no unique relationship between color and orientation, so many observations must be made with the sample in different orientations with respect to the polarized light. This is accomplished on a universal stage, which allows the specimen to be tilted and rotated. By observing the colors at many different orientations, it is possible to determine the orientation of the optical axis. This technique was developed by geologists and is applied primarily to minerals that have relatively large grain sizes and are transparent in thin sections. Samples must be ground to a 
thickness a few tens of microns and polished on both sides. The image analysis that is needed to determine the crystal orientations from these micrographs has been automated and the technique is referred to as computer integrated polarization microscopy, or CIP [26]. By tilting the specimen so that each triple junction is aligned parallel to the viewing direction, it is also possible to accurately measure the directions of the grain boundary planes. Therefore, polarized light microscopy can be used to determine all five macroscopically observable grain boundary parameters.

\subsection{X-ray Methods}

The opacity of many specimens can be overcome by using transmission X-ray imaging. In the simplest mode, grain boundaries in transmission x-ray microradiographs can be visualized if they are marked with a denser material that absorbs $\mathrm{x}$-rays more strongly than the grains. In its original application, two stereoscopic microradiographs were recorded of a $1 \mathrm{~mm}$ thick plate of an Al-Sn alloy so that the grains could be visualized in three-dimensions [27]. In this case, the $\mathrm{Sn}$ wets the $\mathrm{Al}$ grain boundaries to provide the necessary contrast. More recently, a full three-dimensional tomographic reconstruction of an Al-Sn alloy has been made on the basis of 625 microradiographic projections [28]. One advantage of microradiography is that it makes it possible to study kinetic processes within the solid [29].

While microradiographic techniques have allowed the shapes of grains in contact with liquid Sn to be measured, they do not provide information on grain orientations and, therefore, do not allow the measurement of $\square(\square g, \mathbf{n})$. Two closely related techniques, three-dimensional x-ray diffraction (3DXRD) microscopy and the differential aperture $\mathrm{x}$ - 
ray microscope (DAXM), promise to overcome this limitation. The 3DXRDM transmits monochromatic high energy synchrotron radiation through the specimen and records diffracted spots on parallel planar detectors [30-33]. The grain orientation is determined from the angles of the diffracted beams, the shape of the grain section is determined from the shape of the diffracted spot, and the position of the grain within the sample is determined by extrapolating the beam path from the positions where it intersects the detectors. The DAXM is a polychromic Laue transmission technique [34]. Depth resolution is obtained by recording Laue patterns on a planar detector as a knife-edge aperture (in practice, a Pt wire) is moved across the sample. Contrast changes are then used to infer the positions of grains within the bulk of the sample. The spatial resolutions of 3DXRDM and DAXM are in the range of a few microns so they are limited to fairly coarse grained materials. These techniques have the potential to monitor kinetic processes, since they are able to nondestructively measure $\square(\square g, \mathbf{n})$ at different points in the process. However, the emergence of these techniques has been so recent that they have not yet been widely applied.

\subsection{Transmission Electron Microscopy}

Transmission electron microscopy (TEM) is well suited to measuring all five macroscopic grain boundary parameters. Selected area diffraction patterns allow the grain orientations on either side of the boundary to be determined and because images are projections though the specimen, the boundary inclination can also be measured. While TEM is widely used to characterize grain boundaries, one limitation is that samples must be thinned to electron transparency and, therefore, the total area that can be analyzed is 
rather small. This makes the study of large grained materials inconvenient. TEM is most easily applied to specimens where the grain size is large compared to the sample thickness and small compared to the lateral dimensions of the electron transparent region.

In the past, the large number of distinct grain boundary types (see Fig. 4) has made it difficult to survey a significant number of boundaries by TEM. Recently, however, automated procedures for mapping orientations in the TEM have been developed. One technique records electron diffraction patterns at a set of fixed coordinates, indexes the patterns, and then creates an orientation map based on these data [35]. A second technique is to record many dark field images of the same area using a wide range of beam conditions. Each pixel is then analyzed to find the beam conditions that brought it into the diffraction condition and this set of diffraction vectors is used to determine the orientation [36]. These techniques promise to significantly increase the resolution of orientation mapping.

\subsection{Serial Sectioning}

The three dimensional interfacial network can be completely reconstructed on the basis of data from planar sections, a procedure usually referred to as serial sectioning. On a single section plane, we see the lines of intersection between the surface and interfacial network (for example, the upper plane in Fig. 8). If a small amount of material can be removed by polishing or milling ( $\square$ h in Fig. 8), and the second section plane is imaged, then the boundary planes can be interpolated from the first layer to the second. If the procedure is repeated multiple times, the entire three-dimensional microstructure can be reconstructed. There are several obvious requirements and limitations for this 
process. The first is that the amount of material removed between the section planes must be small in comparison to grain size. The second is that it must be possible to align and position the images of each section plane in three-dimensional space. Finally, the line segments that come from the same interface, but appear on different sections, must be identified and connected. A sample of the grain boundary traces in two parallel serial sections is illustrated in Fig. 9 [4].

To determine $\square(\square g, \mathbf{n})$, it is also necessary to measure the crystal orientations. This can be accomplished using electron back scattered diffraction (EBSD) mapping [37]. In a scanning electron microscope (SEM), the sample is inclined with respect to the beam so that a backscattered diffraction pattern can be captured on a planar detector. The pattern can be indexed to determine the local orientation of the crystal at the position where the beam diffracted. By sequentially positioning the beam at a set of predefined positions on the surface, an orientation map can be generated. We note that in this orientation map, four of the five measurements required to determine $\square(\square g, \mathbf{n})$ are specified.

Conventional polishing and milling machines, as well as focused ion beams, have been used to remove thin sections [4, 38-43]. Calibrated serial sectioning using a conventional polishing $[4,38,39]$ or milling machines [40-41] can remove layers with thicknesses on the order of a few microns while the focused ion beam can remove layers with nanometer-scale thicknesses $[42,43]$. Alignment of the sections is usually accomplished using fiducial marks, and the most common are intentionally induced marks from a Vickers hardness indenter [44]. Since the indenter tip leaves marks of known shape, both the alignment of the layers and the vertical position can be 
determined. If the grains do not have a shape anisotropy, then alignment of large areas can be accomplished using the grains [4] or triple junctions [45] as the fiducial marks. In this procedure, the relative position of the each layer is determined by assuming that the positions of the microstructural features (the centers of grains or triple junctions) are randomly distributed about the sample normal.

\subsection{Stereological measurment of $\square \square g, \boldsymbol{n})$}

The analysis of data from a single section plane can be used to derive certain average properties of the interfacial network, but not the actual three-dimensional network configuration. For example, if one wants to measure $\square(\square g, \mathbf{n})$, the information in a single section plane can be used to specify four of the five parameters. The only unknown parameter is the angle between the observation plane and the grain boundary plane. While there is no way to determine this absolutely from the data on a single plane, it is possible to combine many observations from sections of indistinguishable bicrystals to specify the probability that it is a certain plane. The method for deriving $\square(\square g, \mathbf{n})$ from stereological measurements is an extension of the stereology for boundary planes in the sample reference frame originally described by Hilliard [46] and Adams [47]. To measure $\square(\square g, \mathbf{n})$, the analysis must be conducted in the misorientation reference frame described in Section 2.3 [5]. This analysis has been conducted to recover $\square(\mathbf{n})$ for a single misorientation [48] and at all misorientations [49]. Finally, the boundary stereology can also be used to measure $\square(\mathbf{n})$ for crystals of one phase embedded in another [50]. Here, we outline a discrete analysis [49]. 
As a starting point, the five parameter grain boundary space is discretized as described in Section 2.3. The five dimensional matrix of different grain boundary types is referred to as an accumulator. The vector $\mathbf{l}^{\prime}{ }_{i j}$ is defined as the trace where the $\mathrm{j}^{\text {th }}$ triangular boundary tangent plane on the $\mathrm{i}^{\text {th }}$ grain meets the surface, as illustrated in Fig.

1. While each $\mathbf{I}_{i j}^{\prime}$ can be associated with a specific misorientation in the five parameter space, $\mathbf{n}_{\mathrm{ij}}$ is not well defined. However, it must be true that the actual grain boundary plane belongs to a set of planes that includes the surface trace and obeys the condition $\mathbf{l}_{i j}^{\prime} \cdot \mathbf{n}_{i j k}^{\prime}=0$, where the vectors $\mathbf{n}_{i j k}^{\prime}$ are a set of D unit normals to the possible grain boundary planes. Therefore, each observed trace generates a set of D vectors perpendicular to possible boundary planes that can be transformed to the crystal reference frame. With knowledge of the misorientation, the vectors $\mathbf{n}_{i j k}$ specify D cells in the accumulator to which $\left|\mathbf{I}_{\mathbf{i j}}\right|$ is added so that the observations are weighted by line length. In other words, the length of each observed trace is added to the accumulator D times. In the end, we would like to know the fractional length of line segments crossing each plane, since this is equal to the fractional area of each plane [51].

If there are $\mathrm{N}$ observations of traces from indistinguishable bicrystals, then we can be certain that for this misorientation, the accumulator contains $\mathrm{N}$ correct boundary normal orientations and N(D-1) incorrect assignments. To get the line length crossing each boundary type, we have to remove the incorrect assignments from the accumulator. To do this, it must be realized that the incorrect assignments are not distributed randomly. If there is a peak in the real distribution of grain boundary planes, then orientations very close to this pole will have more incorrectly assigned length than others. This is because these neighboring cells have a greater probability of being in the zone of the peak 
orientation than an orientation further away. Conversely, orientations far from the maximum are less likely to share a zone with the most highly populated orientation and will accumulate less incorrectly assigned length. We can describe this inhomogeneous distribution in the following way. In the $p^{\text {th }}$ boundary plane cell for a particular misorientation, there is an observed length, $l_{p}{ }^{0}$. This is the sum of the length correctly assigned to this cell $\left(l_{p}^{c}\right)$ and the length that was correct in some other cell, $q$, but was assigned to $p$ because $p$ and $q$ were both in the zone of the observed trace. Therefore, the total length is:

$$
l_{p}^{o}=l_{p}^{c}+\square_{q=1, q \neq p}^{D^{2}} \square_{p q} l_{q}^{c}
$$

where $\square_{p q}$ is a weighting factor that describes the fraction of great circles whose true plane is $q$, but also pass through $p$ because they are in the same zone. The factor $\square_{p q}$ is largest when $p$ and $q$ are neighboring cells and decreases to a constant as the angular separation between $p$ and $q$ increases. This suggests a method for determining an approximate solution. First, the average value of the incorrectly assigned length that originated from distant (non-nearest neighbor) cells can be estimated and subtracted from from the $p^{\text {th }}$ cell. Second, the incorrectly assigned length that originated in nearest neighbor cells can be estimated and subtracted from this quantity. By making a number of approximations for the sum on the RHS of Eq. 5, one can arrive at the following expression for the correct line length [49]: 


$$
l_{p}^{c}=\frac{l_{p}^{o}+(Z \square 1) \frac{(D \square 1)}{D}\left\langle l^{o}\right\rangle}{1+Z(D \square 1)}
$$

where $Z=2 / D$ and $\left\langle l^{0}\right\rangle$ is the average value of $l^{0}$. When the method is tested against model data sets and data recovered from serial sectioning, satisfactory agreement is obtained (see Fig. 10). The agreement between the stereologically recovered distribution and the true distribution is a function of the number of observations. If $\square(\square g, \mathbf{n})$ is partitioned into $10^{\circ}$ cells and $10^{4}$ grain boundary traces are used, then there are errors of greater than 0.5 MRD in a large fraction of the cells (see Fig. 11). However, if $2.5 \times 10^{5}$ boundaries are measured, then less than $1 \%$ of the cells have an error this large [49].

While information on the local connectivity is not retrieved, the stereological analysis has several advantages for the measurement of $\square(\square g, \mathbf{n})$. The first is that it is not subject to the errors associated with alignment of the images. The second is that the entire process can be automated. Orientation data can be collected by EBSD mapping, and the grain boundary line segments can then be extracted and used for the analysis. Then main disadvantage is the time required to measure a sufficient number of grain boundaries. However, as the speeds of modern EBSD mapping systems increase, this becomes less of a factor. Currently, this is the simplest way to measure $\square(\square \mathrm{g}, \mathbf{n})$.

\subsection{Computer modeling}

Computer modeling has the potential to make a substantial contribution to the study of grain boundary networks. In the past, computational speed limited most simulations to two dimensions. In the last few years, however, calculations based on the 
Monte Carlo Potts model [52], vertex tracking [53,54], boundary tracking [55,56], phase field [57], and gradient-weighted moving finite elements [58] have been used to simulate the structure and evolution of grain boundary networks in three-dimensional systems. To date, the simulations have used only isotropic or highly simplified models for the anisotropic grain boundary properties. However, five parameter grain boundary property models are expected in the near future. The number of distinct grain boundary types (see Fig. 4) means that these simulations must include a large number of grains and it is this aspect that presents the most significant challenge to computing resources.

\section{Observed Distributions of Internal Interfaces}

\subsection{Observed Grain Shape}

The polyhedron that comes closest to filling space with the minimum surface area and meeting the interfacial energy constraints is the tetrakaidecahedron. This 14-sided polyhedron has six faces with four edges and eight faces with six edges and is shown in Fig. 12 [59]. To make this shape meet the surface tension constraints, some curvature must be introduced in the faces with six edges and this will lead to curvature driven boundary motion. Since Matske's [14] observations of 600 bubbles with a uniform size showed that the average number of faces per bubble, $\langle\mathrm{F}\rangle$, is 13.7 and that the average number of edges per grain, $<\mathrm{E}>$, is 5.1, the tetrakaidecahedron seems to be a reasonable approximate model for the average bubble. However, in a polycrystal, there is actually a distribution of grain sizes and shapes; larger grains have more faces and smaller ones have fewer. In two studies of systems of bubbles with mixed sizes (polydispersed), $<$ E $>$ 
has been determined to be 5.1 and $<\mathrm{F}>$ has been measured to be 13.3 and $13.4[15,16]$. In three-dimensional simulations of polycrystals with isotropic boundary properties using the phase field [57], boundary tracking [55,56], Monte Carlo [60] and vertex [54] models, $<\mathrm{F}>$ is cited to be between 13.3 and 13.8 and $<\mathrm{E}>$ to be between 5.01 and 5.07. A smaller value of $<\mathrm{F}>$ obtained from an early Monte Carlo model (12.85) has been attributed to an artifact in the model that permitted nonphysical grain coalescence events $[52,57]$. In summary, therefore, the average topological parameters extracted from experiments and simulations of three-dimensional polydispersed foams (analogous to polycrystals with isotropic interfacial properties) are remarkably consistent.

Experimental results from real polycrystals are less consistent and different from those of foams. Based on the analysis of 100 grains in an Al-Sn alloy observed by stereoscopic microradiography, $<\mathrm{F}>=12.48$ and $<\mathrm{E}>=5.02$ [27]. Hull's [23] more extensive data set on $\square$-brass showed that a histogram of grain diameters has a log normal distribution. Within this distribution, the smallest grains had an average of 8 faces per grain and the largest had 40. For grains in the most highly populated size fraction, the average number of faces per grain was 11.8. The average number of edges per side varied less dramatically with grain size, increasing from four to six from the smallest to largest grains. Faces with five edges have the highest frequency and the average number of edges per face is 4.92 . For 72 grains of $\mathrm{Fe},<\mathrm{F}>$ has been reported to be 13.42 and $<\mathrm{E}>=5.1[60]$. For approximately 1000 grains in a stainless steel, $<\mathrm{F}>$ has been cited to be 14 [24]. Thus, if one compares the results from crystalline grains with those from soap bubbles, we can say that in both cases the average number of edges per face is close 
to five, but that the number of faces on the average grain is less consistent. This inconsistency is evidence that crystalline anisotropy affects topology.

In multiphased materials, shapes can be much more complex. For example, an analysis of cementite precipitates in Fe 1.3 wt \% C 13.4 wt \% Mn showed that grain boundary precipitates exhibited a dendritic structure within the plane of the boundary and that precipitates growing into the grain are thin in one direction and have both lath and plate morphologies [38]. The complex structure of $\mathrm{Sn}$ dendrites in solidified $\mathrm{Pb}-\mathrm{Sn}$ alloys has also been determined by serial sectioning and this has allowed local measurements of both the mean and Gaussian curvatures [40]. It was found that the dendritic structures were well approximated by contiguous cylindrical objects and that $51 \%$ of the internal surface was saddle-shaped. By measuring the orientation of the crystals, it is possible to derive average shape information stereologically. For example, this technique has recently been used to measure the habit of WC crystals in a WC/Co composite [61]. The Co forms $30 \mathrm{vol} \%$ of the material and is liquid at the processing temperature. The WC crystals are fully faceted trigonal prisms bounded on top and bottom by $\{0001\}$ basal facets and on the sides by three $\{10-10\}$ prismatic facets. Although the internal atomic structure of WC is hexagonal, the crystals have trigonal habits because of the material's polar surface chemistry.

\subsection{Observed distribution of internal grain surfaces, $\square(\boldsymbol{n})$}

There has been a tendency for experimental studies to concentrate on the misorientation of grain boundaries rather than the grain boundary plane. Nevertheless, there are numerous qualitative observations that indicate the preference for various 
materials to favor the formation of grain boundaries on specific planes; we begin by reviewing some examples. Nonmetallic compounds are typically more anisotropic than metals and flat, low index grain boundary planes are usually more obvious. For example, polarized light microscopy studies showed that grain boundary planes in polycrystalline aggregates of amphibole, biotite, and pyroxene (all silicate minerals) are usually configured so that one of the adjacent grains is terminated by a low index facet [62-64]. TEM observations have shown that symmetric tilt grain boundaries in $\mathrm{NiO}$ [65] and $\mathrm{SrTiO}_{3}[66]$ favor asymmetric configurations where one of the two crystals is terminated on a $\{100\}$ plane. The preference for certain grain boundary planes in ceramics is especially clear in cases where the grains grow abnormally large and/or there is an intergranular liquid phase present at the processing temperature. Flat asymmetric grain boundaries with $\{111\}$ habit planes are observed in titania-excess $\mathrm{BaTiO}_{3}[67]$ and facets with $\{100\}$ orientations are revealed by $\mathrm{PMN} / \mathrm{PT}$ grains growing in a $\mathrm{PbO}_{2}$ rich intergranular liquid $[68,69]$. In some ranges of temperature and composition, grain boundaries in $\mathrm{Al}_{2} \mathrm{O}_{3}$ also show a tendency to favor certain low index planes [70, 71].

Some investigations have revealed that grain boundaries in metals also prefer low index planes. For example, tilt boundaries in gold show a preference for densely packed low index planes such as $\{100\},\{111\},\{112\}$ and $\{110\}[72,73]$. For $[100]$ tilt boundaries in $\square-\mathrm{Fe},\{110\}$ planes are observed to be special so that asymmetric tilt boundaries with one crystal terminated by $\{110\}$ are favored over symmetric configurations [74]. A tendency for certain high index boundary planes has also been cited. For example, $\{113\} \|\{335\}$ and $\{117\} \|\{551\}$ boundaries have been reported to be common in $\mathrm{Cu}[75]$ and $\mathrm{Al}[76,77]$, respectively. However, it should be noted that these 
boundaries are the incoherent components of low index twin boundaries and might be necessary to meet topological requirements. It has been hypothesized that certain boundary planes are preferred because these geometries place a relatively high fraction of the sites on the surface of one crystal in coincidence with those on the surface of the adjacent crystal. However, based on their analysis of [100] and [110] tilt boundaries in Au, Goodhew et al. [72] concluded that the planar coincidence does not provide a satisfactory explanation for the preference for certain planes.

Recently, more comprehensive measurements of $\square(\mathbf{n})$ have been reported for $\mathrm{MgO}$ [4], $\mathrm{SrTiO}_{3}$ [78], $\mathrm{MgAl}_{2} \mathrm{O}_{4}, \mathrm{TiO}_{2}$, and $\mathrm{Al}$ [79]. The result for $\mathrm{MgO}$ has already been shown in Fig. 3 and the results for the other materials are given in Fig. 13. The tendency for grain boundaries in $\mathrm{MgO}$ to form on $\{100\}$ planes is thought to be related to its surface energy anisotropy, $\square(\mathbf{n})$. The favored planes, $\{100\}$, have the lowest surface energy and those that occur with the lowest frequency, $\{111\}$, have the highest surface energy $[80,81]$. The distribution of grain boundary planes in $\mathrm{SrTiO}_{3}$ is also well correlated to the surface energy ansiotropy. The most common grain boundary plane, $\{100\}$, is also the lowest energy surface plane [82-84].

For the case of $\mathrm{MgAl}_{2} \mathrm{O}_{4}$, the data show that internal $\{111\}$ planes occur with more than twice the frequency of $\{100\}$ planes. While the surface energy anisotropy has not yet been measured for this material, the boundary plane preference agrees with the habits of free surfaces of naturally occurring spinels, which are typically dominated by $\{111\}$ planes [85]. The most frequently observed twin and cleavage plane in spinel is also (111). Surface energy calculations, on the other hand, have yielded ambiguous results [86, 87]. Early calculations concluded that the (111) plane has the lowest energy 
[86], while more recent calculations indicate a minimum at the (100) orientation [87]. Even so, the habits of the grains in the polycrystal are consistent with the habits of spinel crystals observed in natural settings.

Tetragonal $\mathrm{TiO}_{2}$ (rutile) crystals display $\{110\},\{100\},\{101\}$, and $\{111\}$ facets. The cleavage planes are $\{110\}$ and $\{100\}$ and twins are found on $\{101\}$ planes [88]. Calculations identify the minimum energy surface as (110), while the (100) and (101) surfaces have higher energies [89]. The internal surfaces of the rutile polycrystal show the same trend (see Fig. 13c): $\{110\}$ planes are the most abundant, followed by $\{100\}$, $\{111\}$, and $\{101\}$. It is noteworthy that the minimum of the distribution in this tetragonal material occurs at the (001) orientation, consistent with its absence on growth habits and the theoretical result that it has the highest energy [89].

The cohesive forces in $\mathrm{MgO}, \mathrm{SrTiO}_{3}, \mathrm{MgAl}_{2} \mathrm{O}_{4}$, and $\mathrm{TiO}_{2}$ are dominated by long range electrostatic interactions. Aluminum was examined as an example of a crystalline material with short range cohesive interactions. Here, internal $\{111\}$ planes are preferred. The (111) surface has the highest coordination number of any plane in this structure and observations of microscopic cavities in aluminum suggest that this is the plane of minimum energy [90]. In all of the cases that have been examined, there seems to be a clear connection between the energies of the free surfaces and the planes that bound the internal interfaces. This relationship is also reflected in the magnitude of the deviation from random in the distributions of internal surfaces. The maximum and minimum values of $\square(\mathbf{n})$ for $\mathrm{Al}$ (see Fig. 13d), which is thought to have the smallest surface energy anisotropy of the materials studied, deviate less from random than the other materials. Thus, the overall conclusion that can be drawn from these observations 
is that the habit of a phase in contact with itself in a dense polycrystal (but misoriented) is not substantially different from its habit when in contact with a gas or liquid phase.

While there appears to be a clear connection between $\square(\mathbf{n})$ and $\square(\mathbf{n})$, it should be remembered that while $\square(\mathbf{n})$ is an equilibrium quantity, $\square(\mathbf{n})$ is the result of grain growth, which is a kinetic process. Therefore, the observed average grain habits should be thought of as growth forms rather than equilibrium shapes. So, while it would not be appropriate to assume an exact inverse correlation between the observed distribution of planes and the surface energy anisotropy, we should also recognize that the slow growing faces found on crystal habits typically correspond to low energy surfaces and, in this way, we can explain the tendency for certain low energy planes to be preferred on grain shapes.

Although the peaks in $\square(\mathbf{n})$ show that grains in polycrystals display preferred habit planes, the topology of the network demands the simultaneous presence of nonhabit planes. For example, since the average number of faces on a grain (12-14) is greater than the multiplicity of the habit planes, and these planes have some curvature, it is necessary to introduce non-habit planes in the interfacial network. Furthermore, if there is a low index plane on one side of the boundary, then the plane on the other side is determined by the lattice misorientation and for an arbitrary misorientation, the adjoining surface is most likely to be a non-habit plane. Because of these geometric constraints, it would be incorrect to think of the polycrystal as a collection of self-similar shapes. 


\subsection{Observed grain boundary character distribution, $\square(\square g, \boldsymbol{n})$}

When the microstructure is viewed at a collection of individual grains, as described above, the grain-to-grain relationships are ignored. In fact, the lattice misorientation is the most frequently used parameter to characterize grain boundaries. It has been common practice to separately distinguish low misorientation angle $\left(\square<15^{\circ}\right)$ boundaries from high misorientation angle boundaries and special boundaries. Special boundaries are those with a special coincidence relationship, where a certain fraction of the sites in the adjacent lattices are in coincidence. The special boundaries are named by the inverse of the number of coincident sites. For example, the $\square 3$ misorientation in a cubic crystal is a $60^{\circ}$ rotation around [111] and leads to a configuration in which every third lattice site is coincident. Higher $\square$ numbers signify a decreasing concentration of coincident sites. The lattice coincidence is most likely to have physical significance only when it occurs in the boundary plane. Thus, while CSL boundaries are usually characterized only by $\square g$, the condition of high coincidence is more restrictive; for a specific CSL misorientation, high atomic coincidence at the interface occurs only at a few specific values of $\mathbf{n}[91]$.

There are numerous examples in which the low $\square$ CSL boundaries appear to be special in their population and properties. For example, polycrystals with high fractions of these boundaries have been shown to have properties that are superior to materials with more random grain boundary networks $[92,93]$. In most cases, the fraction of special boundaries is evaluated based only on misorientation measurements. Therefore, it is not clear what fraction of the low sigma CSL boundaries are on planes of high coherency. Recent measurements of the distribution of grain boundary planes for $\square 3$ 
boundaries have shown that while most are (111)\|(111) $60^{\circ}$ twist boundaries (the socalled coherent twin), there is also a measurable population of incoherent boundaries [ 94 , 95]. The role of these incoherent boundaries in determining the properties of the material has not yet been clarified.

In experiments where the interface plane is controlled or measured, the influence of lattice coincidence on the energy, $\square \square \mathrm{g}, \mathbf{n})$, is more clear. Grain boundary dihedral angles at thermal grooves or triple junctions are widely used to evaluate relative boundary energies and have shown that in some cases low $\mathrm{CSL}$ boundaries have relatively low energies if the planes have high coherency [96-105]. For example, Kimura et al. [102] and Dhalenne et al. $[100,101]$ demonstrated that the energies of $<110>$ symmetric tilt boundaries with a high degree of coincidence were relatively lower than those that did not. However, this is true only for interface normals with a high degree of planar coincidence. Rotations of $70.53^{\circ}$ and $109.47^{\circ}$ about [110] are both $\square 3$ misorientations; however, a high degree of coincidence in the intergranular region occurs only for symmetric $\{111\}$ boundary planes. This configuration is realized by a symmetric $70.53^{\circ}$ rotation, but not for the symmetric $109.47^{\circ}$ rotation. In the case of $\mathrm{MgO}$, the energy of $109.47^{\circ} \square 3$ rotation was approximately $40 \%$ higher than the $70.53^{\circ}$ $\square 3$ rotation [102]. The effect is even larger in the case of $\mathrm{NiO}$, where the energy of the $109.47^{\circ} \square 3$ rotation is $300 \%$ higher than the $70.53^{\circ} \square 3$ rotation $[100,101]$. Therefore, we can surmise that the boundary plane has a significant influence on the energy and that any special properties that might be associated with the CSL also vary with $\mathbf{n}$. Further evidence for the relatively low energies of grain boundaries with high coincidence interface planes comes from the observation that spherical metal crystals on flat surfaces 
rotate to positions of high coincidence [106-107] and that aggregates of $\mathrm{MgO}$ smoke have extraordinarily high fractions of low $\mathrm{CSSL}$ twist configurations [108]. Finally, it should be noted that there are also observations showing that some low $\mathrm{CSL}$ boundaries (boundaries with high coincidence) do not have reduced energies [96-98, 100].

Until recently, most grain boundary studies have concentrated on small segments of the five dimensional space with highly symmetric boundaries that can have a high concentration of coincident sites in the interface. It is now possible to make more comprehensive measurements of $\square(\square g, \mathbf{n})$ using the techniques described in Section 3 $[4,78,109]$. The results have shown that at specific misorientations, the anisotropy in the distribution of grain boundary normals is large in comparison to the distribution of internal grain surfaces $(\square(\mathbf{n}))$.

One interesting aspect of the grain boundary normal distributions at fixed misorientations is that because grain surfaces favor certain low index orientations, asymmetric boundaries must also be favored. As long as there is a low index plane on one side of the boundary, then the plane on the other side is determined by the lattice misorientation; if the misorientations are distributed randomly, the surface of the adjoining grain is not likely to be another low index plane. For example, Fig. 14 illustrates how the distribution of grain boundary planes changes with misorientation angle for $\mathrm{MgO}$ boundaries with [100] misorientation axes [4]. At each misorientation angle, a peak in the distribution occurs at a $\{100\}$ type plane. While these are the only peaks in the distribution at relatively low angles $\left(5^{\circ}\right.$ and $\left.15^{\circ}\right)$, the distribution broadens at higher angles $\left(25^{\circ}\right)$ and eventually multiple peaks emerge $\left(35^{\circ}\right)$. These changes are easy to understand if one considers the strong preference for $\{100\}$ type planes and the 
geometric constraints associated with creating a bicrystal. For $\{100\}$ twist boundaries, both crystals can be terminated by a $\{100\}$ plane. Thus, we notice that the distribution of grain boundary planes around the twist positions (see Fig. 14) do not change significantly with misorientation angle. However, for pure tilt boundaries, if one of the terminating planes is fixed at $\{100\}$, the complementary plane in the adjoining crystal must have a different index. Therefore, we see that along the zone of pure tilt boundaries, the population varies systematically. If one crystal is terminated by (001), then the complementary crystal must be terminated by a $(0 \mathrm{k} 1)$ plane inclined by the misorientation angle from (001). Thus, the peak at (001) spreads along the zone of the tilt boundaries as the misorientation angle increases and, at $35^{\circ}$, forms a separate peak inclined from (001) by this same angle.

The tendency to form grain boundaries with low index planes can be compared to the tendency to form low $\square$ CSL boundaries with high planar coincidence. Figure 15 shows the population and energy of pure tilt $\square 5$ boundaries in $\mathrm{MgO}$ [110]. The boundaries that couple the highest population and the lowest energy are asymmetric tilt boundaries of the type $\{100\} \|\{430\}$. At the positions of the coherent, high coincidence symmetric tilts, $\{120\} \|\{120\}$, the population reaches a minimum and the energy reaches a maximum. This indicates that asymmetric boundaries with $\{100\}$ planes are favored over the more symmetric high coincidence boundaries. This is consistent with TEM observations of symmetric $\square 5$ tilt bicrystals in $\mathrm{NiO}$ [65]. The other symmetric boundary in this tilt system, which has only partial coincidence, is the $\{310\} \|\{310\}$ configuration. This boundary has an energy similar the asymmetric $\{100\} \|\{430\}$ configuration, but occurs at a minimum in the population. 
Similar results were found in $\mathrm{SrTiO}_{3}$ [78]. The distributions of grain boundary planes at the four lowest $\square$ CSL misorientations are illustrated in Fig. 16. In each case, the interfaces of highest planar coincident site density are the pure twist boundary (plane normal and misorientation axis parallel) and the symmetric tilt boundaries (when the misorientation axis is in the boundary plane and the surfaces on either side of the boundary are the same) that are marked on the stereograms. For the $\square 3$, we see a maximum in the vicinity of the pure twist boundary formed by two (111) planes rotated by $60^{\circ}$. In the results from $\mathrm{SrTiO}_{3}$, this is also the only example of where a boundary formed by two high energy surface planes is a local maximum in the population. This probably occurs because of the remarkably high coherency across this special boundary. Note that minima are found at the positions of the $\{211\}$-type symmetric tilt boundaries, which have next highest concentration of coincident sites in the interface plane.

For the $\square 5, \square 7$, and $\square 9$ boundaries, on the other hand, the peaks are instead correlated with boundaries that have planes near $\{100\}$ and the complements to these surfaces; there are no distinct maxima at the planes of high coincidence. One apparent exception is the (100)॥(100) twist boundary at the $\square 5$ position. However, this maximum occurs at all [100] misorientations and is better explained by the presence of the two low energy planes than by the coincidence condition [78]. In the interpretation of these data, it should be noted that because misorientation space has been discretized in approximately $10^{\circ}$ increments, the plots in Fig. 16 average the distribution of grain boundary planes at each CSL misorientation with those of neighboring misorientations within a $10^{\circ}$ window. Thus, if there are cusps in the distribution at the CSL misorientations, the true population will be diluted by the coarse discretization. The 
effect is that extreme values move closer to the average. Thus, while the discretization may cause us to underestimate the actual values at the extreme positions in the distribution, it doesn't alter the basic conclusion that for misorientations other than $\square 1$ and $\square 3$, the coherent boundary planes that do not have the $\{100\}$ orientation are local minima in the population.

Assuming that this preference for certain grain boundary planes is a reflection of the boundary's properties, then this is a potentially valuable avenue for controlling the properties of materials that depend on the grain boundary network. So-called grain boundary engineering efforts currently attempt to improve the properties of materials by increasing the fraction of low $\square$ CSL boundaries with coherent planes [92, 93]. Recall that CSL boundaries with high planar coincidence occur at only selected points in the five parameter space. Low index planes, on the other hand, can be realized for boundaries of any misorientation. Therefore, there are many more of these boundaries than the special CSL boundaries. The influence of these special planes on properties is not yet known.

It is also now apparent that there is a connection between the grain boundary population, $\square(\square g, \mathbf{n})$, and the energy $\square \square g, \mathbf{n})$. It has long been assumed that there is an inverse correlation between the two quantities. In the case of $\mathrm{MgO}$, the relative grain boundary energy, $\square \square g, \mathbf{n})$, has been determined by analyzing the dihedral angles of the triple junctions $[109,110]$. As illustrated in Fig. 17, there is, in fact, a strong correlation between the grain boundary energy and the population. Boundaries with high relative energy are observed less frequently than boundaries with a low relative energy.

As noted in the Section 4.2, there is also an apparent correlation between the surface energies and the population and this implies a correlation between the free 
surface energy, $\square(\mathbf{n})$, and grain boundary energy, $\square(\square g, \mathbf{n})[78,79,109,110]$. This is not too surprising, since both quantities reflect the local disruption in bonding at the interface. If we imagine creating a grain boundary by first creating the two free surfaces and then joining them, we can say that the boundary energy is the sum of the two surface energies, minus a binding energy that results from the interactions of the atoms on either side of the interface [111]. As a first order approximation, we can take the binding energy to be constant and use the sum of the two surface energies that make up the boundary as hypothetical grain boundary energy. The data in Fig. 18 and 19 illustrate that the sum of the two surface energies is correlated to the grain boundary population; the most abundant boundaries in $\mathrm{SrTiO}_{3}$ are those that are composed of relatively low energy surfaces [78].

The observed correlation between the grain boundary and surface energies indicates that either the binding energy is somehow related to the surface energy, or that it is more or less constant. Theoretical estimates have shown that the magnitude of the binding energy increases with the average interplanar spacing of the two surfaces adjoining the boundary [112-114]. Since the lowest energy surface planes are those that break the fewest bonds, and these correspond to the densest planes that also have the largest interplanar spacing, we can assume that the surface energies vary inversely with the interplanar spacing. So, for a boundary comprised of two low index surfaces, the two surface energies are relatively low and the binding energy is maximized, an effect that leads to a minimum in grain boundary energy for this configuration. Conversely, two high index, high energy surfaces will have a smaller binding energy and represent a maximum in the grain boundary energy. In fact, for high index surfaces with small 
interplanar spacings, the binding energy is nearly constant. This is because of the incommensurate structure in the interface plane. Boundary atoms in such interfaces experience a wide range of possible atomic configurations. As long as the details of the atomic relaxations in a specific boundary are as widely varied along the aperiodic structure as they are from boundary to boundary, they average to about the same value. Thus, because the binding energy changes systematically with d-spacing and is relatively constant for high index surfaces, the surface energies provide a good measure of the density of unsatisfied bonds in the interface and, therefore, the grain boundary energy.

Finally, we should note that there are special cases where strong variations in the interface coincidence will lead to significant increases in the binding energy that may not follow the same trend as the surface energies. For example, low angle boundaries are better visualized as periodic dislocation arrays and in these cases, the surface energy is not expected to provide a good model for the grain boundary energy. An analysis of low angle grain boundaries in $\mathrm{MgO}$ [110] showed that conventional theories [115, 116] provide a satisfactory explanation for the observations. Another example is the coherent twin in $\mathrm{SrTiO}_{3}$. Every atom in the (111)\|(111) $60^{\circ}$ boundary is in a coincident position, so the fact that the (111) plane has a relatively high surface energy is compensated by an unusually large binding energy. As illustrated in Fig. 16a, the boundary still has a relatively high population and, presumably, a low energy.

To close this section, we note that the most appealing feature of the correlation between surface energy, grain boundary energy, and population is that the surface energy is only a two parameter function and, in comparison to the five parameter grain boundary energy, is more easily measured. Therefore, if the correlation between these quantities 
proves to be a general trend in polycrystalline materials, then it has the potential to greatly accelerate our understanding of grain boundary anisotropy.

\subsection{Origins of Interface Distributions}

It seems natural to assume that the anisotropy in $\square(\square g, \mathbf{n})$ is related the anisotropy of the grain boundary properties. However, the mechanisms at work are not clear at this time. The inverse correlation between energy and population in well-annealed polycrystals implies that as grain growth proceeds, higher energy boundaries are eliminated preferentially so that lower energy boundaries persist and dominate the population. However, the idea that the grain boundary population is refining toward a lower energy can be misleading, since no grain boundaries are likely to persist throughout the entire grain growth process; whenever a grain disappears, new boundaries appear. For example, as the grain size increases from 1 micron to 100 microns, only 1 in $10^{6}$ grains survive and the probability that any boundary between two crystals in the initial configuration survives this process is extraordinarily small. The inverse correlation of the population and energy might also be explained as a kinetic phenomenon. Therefore, the time that any given grain boundary exists in microstructure is, on average, equal to the time it takes to move through a neighboring grain of average size. High mobility boundaries move through neighbors in a shorter time interval and are, therefore, annihilated more rapidly than low mobility boundaries. Assuming energy and mobility to be correlated, then, at any instant in time, there should be more low energy (mobility) boundaries than high energy (mobility) boundaries. 
The relative effects of energy and mobility have been explored in recent two dimensional grain growth simulations $[117,118]$. Holm et al. [117] used simulations to show that during growth, the population of grain boundaries with relatively low energies increases and the population of high energy boundaries decreases. During the simulation, the boundary distribution rapidly reaches a steady state and this state is not influenced by the anisotropy of the boundary mobility. Similar phenomena were reported by Upmanyu et al. [118], who also argued that grain boundary energy is the dominant anisotropy in two-dimensional systems. These results were best explained by a model that increases the length of low energy boundaries and reduces the length of high energy boundaries to satisfy the interfacial equilibrium requirement at the triple points. We refer to this as the equilibrium boundary repositioning process. Note that this process does not affect the number density of the different types of boundaries, it simply makes low energy boundaries longer and high energy boundaries shorter. This prediction can be experimentally tested.

While the grain boundary distribution is measured as a per unit area population, it is also possible to examine the number density of the boundaries. In other words, the boundaries can be counted by type, without normalizing by the length or area of each particular segment. When analyzed in this way, we find that the number density of boundaries is always less anisotropic than the area normalized distribution; this indicates that the most commonly observed boundaries have larger average areas, as predicted by the model for the equilibrium boundary repositioning process. However, the remaining anisotropy is significant (approximately $2 / 3$ of the total). In other words, not only are low energy boundaries larger on average than high energy boundaries, but there are more 
of them. The increased number density of low energy boundaries suggests that there is at least one additional mechanism at work. One possibility is that the grain boundary energy and mobility are related, so that higher energy boundaries have higher velocities. If so, the faster boundaries move through neighboring grains at a higher rate and undergo more frequent annihilation. This can lead to an enhancement in the population of low energy boundaries with respect to higher energy boundaries.

\section{Conclusions and Open Questions.}

\subsection{The structures of grain boundary networks}

The coupling of automated EBSD mapping with serial sectioning and new stereological procedures has made it possible to collect statistically significant samples of the distribution of grain boundaries in dense polycrystals. Three-dimensional X-ray diffraction microscopy and three-dimensional computer simulations have the potential to provide important kinetic information, but these capabilities are only emerging now. Grains are volumes of constant orientation with approximately planar faces that meet at edges where there is an abrupt change in orientation. The distribution of grain diameters is approximately log normal and in this distribution, the average grain has 12 to 14 faces and 5.1 edges per face. The crystallographic distribution of the internal grain surfaces, $\square(\mathbf{n})$, is coupled to the surface energy anisotropy, $\square(\mathbf{n})$. Surfaces with low energy are the most likely to terminate internal grain surfaces. The results suggest that the habit of a phase in contact with itself in a dense polycrystal (but misoriented) is not substantially different from its habit when in contact with a gas or liquid phase. 
In two systems that have received the most detailed scrutiny $\left(\mathrm{MgO}\right.$ and $\left.\mathrm{SrTiO}_{3}\right)$ the five parameter grain boundary character distribution, $\square(\square g, \mathbf{n})$, is inversely correlated to sum of the energies of the surfaces that make up the boundary. In both cases, the system favors asymmetric boundaries in which one of the surfaces adjacent to the boundary is a low energy and low index surface. In both cases, the distribution is more sensitive to the boundary plane orientation than it is to the misorientation. With the exception of $\square 1$ and $\square 3$ coherent twins, there was no obvious preference for high coincidence boundaries. Instead, the boundaries that should be considered special with respect to their population are those terminated by low index grain boundary planes, which occur at every misorientation.

\subsection{Challenges for the future}

There are still many aspects of grain boundary networks that are not understood and many opportunities for continued research. First, there are challenges in the areas of angular and spatial resolution. The most comprehensive measurements of $\square(\square g, \mathbf{n})$ have been made at a resolution of $10^{\circ}$. As mentioned before, it is therefore reasonable to expect that sharp features in the distribution have been underestimated and it is possible that some features are missed altogether. Increasing the resolution from $10^{\circ}$ to $5^{\circ}$ is a matter of collecting $2^{5}$ times as much data. This will become easier as the speed of EBSD mapping increases. To increase beyond this point, it will be necessary to carefully consider the accuracy of both the orientation measurements and the beam positioning within the microscope. The current measurements are also somewhat limited in their spatial resolution. The grains must be significantly larger the SEM spot size, but small 
enough that there are enough grains in the sample. It is easy to imagine that when the automated mapping in the TEM is more routine and combined with FIB, it will be possible to measure $\square(\square g, \mathbf{n})$ in grains with nano-scale dimensions. This is a particularly interesting avenue for research, since there is some evidence that grain boundaries in nanoscale materials behave differently than those with microscale dimensions.

The current measurements also suggest that there is a mechanistic link between grain boundary properties, such as energy and mobility, and the grain boundary distribution, $\square(\square g, \mathbf{n})$. If this is so, then it should be possible, for example, to determine $\square \square g, \mathbf{n})$ from measurements of $\square(\square g, \mathbf{n})$. However, to solve this problem, it will first be necessary to understand the mechanisms relating the grain boundary properties of the population and this is where mesoscopic computer simulations of grain growth in threedimensions, with boundary properties that depend on all five parameters, are expected to have an important impact.

The role of low index planes in the macroscopic properties of materials is another interesting area for continued research. Efforts in the area of grain boundary engineering have strived to increase the concentration of low $\square$ CSL boundaries in polycrystals, because this was observed to lead to a variety of improved properties. In this process, the role of the grain boundary plane has not yet been clarified. It should also be noted that the non-CSL boundaries, which are usually ignored, also affect the bulk properties, especially if so-called general boundaries terminated by low index planes have special properties. Finally, the connectivity of the boundaries and correlations between different boundary types is likely to be important to at least some properties, but this area has received very little attention. 


\section{Acknowledgements}

This work was supported by the MRSEC program of the National Science Foundation under Award Number DMR-0079996.

\section{References}

[1] C. Goux, Can. Metall. Q. 13 (1974) 9.

[2] D. Harker and E.R. Parker, Trans. A.S.M. 34 (1945) 156.

[3] H.-J. Bunge, Texture Analysis in Materials Science, Butterworths, London, U.K., (1982) p. 21.

[4] D.M. Saylor, A. Morawiec, and G.S. Rohrer, Acta Mater. 51 (2003) 3663.

[5] A. Morawiec, in: H. Weiland, B.L. Adams, A.D. Rollet (Eds.) Proceedings of the Third International Conference on Grain Growth. TMS, Warrendale, PA (1998) 509.

[6] A.P. Sutton and R.W. Balluffi, Interfaces in Crystalline Materials, Clarendon Press, Oxford, UK, (1995) chapter 5.

[7] F.C. Frank, Met. Trans. 19A (1988) 403.

[8] D. Wolf and J.F. Lutsko, Z. Kristallogr. 198 (1989) 239.

[9] M. Takashima, A.D. Rollett, and P. Wynblatt, Phil. Mag. 80 (2000) 2457.

[10] M. Takashima, P. Wynblatt, and B.L. Adams, Interface Science 8 (2000) 251.

[11] R.C. Pond and W. Bollman, Roy. Soc. London Phil. Trans. 292 (1979) 449.

[12] G. Kalonji and J.W. Cahn, J. de Physique 43 (1982) C6-25.

[13] C.S. Smith "The Shapes of Metal Grains, with some Other Metallurgical Applications of Topology,” Metal Interfaces, (ASM Cleveland 1952) p. 65. 
[14] E.B. Matske, Am. J. of Botany 33 (1946) 58.

[15] E.B. Matske and J. Nestler, Amer. J. of Botany 33 (1946) 130.

[16] C. Monnereau and M. Vignes-Adler, Phys. Rev. Lett. 80 (1998) 5228.

[17] J.W. Marvin, Amer. J. of Botany 26 (1939) 280.

[18] W. W. Mullins, J. Appl. Phys. 27 (1956) 900.

[19] C. Herring, in: W.E. Kingston (Ed.), The Physics of Powder Metallurgy, McGraw Hill, New York (1951) 143.

[20] C.H. Desch, J. Inst. Metals 11 (1914) 57.

[21] C.H. Desch, J. Inst. Metals 22 (1919) 241.

[22] F.C. Hull, Mater. Sci. Technol. 4 (1988) 778.

[23] C.S. Smith, Metal. Rev. 9 (1964) 1.

[24] K. Matsuura, Y. Itoh, T. Ohmi, and K. Ishii, Mater. Trans. JIM 35 (1994) 247.

[25] F.N. Rhines and B.R. Patterson, Met. Trans. A 13 (1982) 985.

[26] R. Heilbronner and C. Pauli, J. Struct. Geol. 15 (1993) 369.

[27] W.M. Williams and C.S. Smith, J. Metals (Trans. AIME) 4 (1952) 755.

[28] C.E. Krill III, K. Dorbrich, D. Michels, A. Michels, C. Rau, T. Weitkamp, A. Snigirev, and R. Birringer, in: U. Bonse (Ed), Developments in X-ray Tomography III, Proc. SPIE vol 4503, SPIE Press, Bellingham, WA (2002) 205.

[29] W.J. Boettinger, P.W. Voorhees, R.C. Dobbyn, and H.E. Burdette, Metall. Trans. A 18A (1987) 487.

[30] D. Juul Jensen, Å Kvick, E.M. Lauridsen, U. Lienert, L. Margulies, S.F. Nielsen, H.F. Poulsen, Mat. Res. Soc. Symp. Proc. 590 (2000) 227. 
[31] S.F. Nielsen, W. Ludwig, D. Bellet, E.M. Lauridsen, H.F. Poulsen, D. Juul Jensen, N. Hansen, X. Huang, D. Juul Jensen, E.M. Lauridsen, T.J. Sabin, and J.A. Wert (Eds.), Proceedings of the $21^{\text {st }}$ Risoe International Symposium on Materials Science: Recrystallization - Fundamental Aspects and Relations to Deformation Microstructure, Risoe National Laboratory, Roskilde, Denmark (2000) 473.

[32] H.F. Poulsen, S.F. Nielsen, E.M. Lauridsen, S. Schmidt, R.M. Suter, U. Lienert, L. Margulies, T. Lorentzen and D. Juul Jensen, J. Appl. Cryst. 34 (2001) 751.

[33] X. Fu, H.F. Poulsen, S. Schmidt, S.F. Nielsen, E.M. Lauridsen, D. Juul Jensen, Scripta Mater. 49 (2003) 1093.

[34] B.C. Larson, W. Wang, G.E. Ice, J.D. Budai, and J.Z. Tischler, Nature 415 (2002) 887.

[35] J.J. Fundenberger, E. Bouzy, A. Morawiec, and J.S. Lecomte, Mater. Sci. Forum 408-12 (2002) 209.

[36] R. Penelle, T. Baudin, D. Dingley, M. Tiner, and S. Wright, Mater. Sci. Forum 408$12(2002) 523$.

[37] B.L. Adams, S.I. Wright, K. Kunze, Met. Trans., 24A (1993) 819.

[38] M. Lanzagorta, M.V. Kral, J.E. Swan II, G. Spanos, R. Rosenberg, E. Kuo, IEEE Visualization (1998) 487-490.

[39] V. Randle and H. Davies, Ultramicroscopy 90 (2001) 153.

[40] J. Alkemper and P.W. Voorhees, Acta Mat. 49 (2001) 897.

[41] J. Alkemper and P.W. Voorhees, J. Microscopy 201 (2001) 388.

[42] D.N. Dunn and R. Hull, Appl. Phys. Lett. 75 (1999) 3414.

[43] D.N. Dunn, G.J. Shiflet, and R. Hull, Rev. Sci. Instrum. 73 (2002) 330. 
[44] V. Randle and O. Engler, Introduction to Texture Analysis: Macrotexture, Microtexture, \& Orientation Mapping, Gordon and Breach Science Publishers, Amsterdam (2000) 270.

[45] A. Morawiec and D. Saylor in: J. Spunzar (Ed.) Proceedings of ICOTOM-12, Montréal, Canada, (1999) 198-203.

[46] J.E. Hilliard, Trans. Metall. Soc. AIME 224 (1962) 201.

[47] B.L. Adams, Met. Trans. A 17 (1986) 2199.

[48] R.J. Larsen and B.L. Adams in: A.S. Khan and O. Lopez-Pamies (Eds), Proceedings of Plasticity '02, Neat Press, Fulton MD, (2002) 27.

[49] D.M. Saylor, B.S. El-Dasher, B.L. Adams, G.S. Rohrer, Metall. Mater. Trans., in press.

[50] D.M. Saylor, G.S. Rohrer, J. Amer. Ceram. Soc. 85 (2002) 2799.

[51] M.G. Kendall and P.A.P. Moran, Geometrical Probability, Hafner Publishing Company, New York, NY, (1963) pp. 78-80.

[52] M.P. Anderson, G.S. Grest, and D.J. Srolovitz, Phil Mag B 59 (1989) 293.

[53] K. Fuchizaki, T. Kusaba, and K. Kawasaki, Phil. Mag. B 71 (1995) 333.

[54] D. Wegand, Y. Brechet, J. Lepinoux, W. Gust, Phil. Mag. B 79 (1999) 703.

[55] F. Wakai, N. Enomoto, H. Ogawa, Acta Mater. 48 (2000) 1297.

[56] F. Wakai, Y. Shinoda, S. Ishihara, A. Dominguez-Rodriguez, J. Mater. Res. 16 (2001) 2136.

[57] C.E. Krill III and L.-Q. Chen, Acta Mater. 50 (2002) 3057.

[58] A. P. Kuprat, SIAM J. Sci. Comput. 22 (2000) 535.

[59] W. Thompson, Phil. Mag. 24 (1887) 503.

[60] G. Liu, Haibo Yu, X. Song, X. Qin, Mater. Design 22 (2001) 33. 
[61] C.-S. Kim and G.S. Rohrer, Interface Science 12 (2004) in press.

[62] R. Kretz, Journal of Petrology 7 (1966) 68.

[63] J. Vernon Canadian Petroleum Soc. 9 (1968) 1.

[64] D. Laporte and A. Provost, Journal of Geophysical Research, 105 (2000) 25937.

[65] K.L. Merkle and D.J. Smith, Ultramicroscopy 22 (1987) 57.

[66] S.B. Lee, W. Sigle, W. Kurtz, M. Rühle, Acta Mater. 51 (2003) 975.

[67] S. B. Lee, W. Sigle, and M. Rühle, Acta Mater. 50 (2002) 2151.

[68] T. Li, A.M. Scotch, H.M. Chan, M.P. Harmer, S. Park, T.R. Shrout, J.R. Michael, J. Am. Ceram. Soc. 81 (1998) 244.

[69] J.S. Wallace, J.-M. Huh, J.E. Blendell, C.A. Handwerker, J. Am. Ceram. Soc. 85 (2002) 1581.

[70] C. W. Park, D. Y. Yoon, J. E. Blendell, and C. A. Handwerker, J. Am. Ceram. Soc. $86(2003) 603$.

[71] M. A. Gülgün, V. Putlayev, and M. Rühle, J. Am. Ceram. Soc. 82 (1999) 1849.

[72] P.J. Goodhew, T.Y. Tan, and R.W. Balluffi, Acta Met. 35 (1978) 557.

[73] H. Ichinose and Y. Ishida, Phil. Mag. A 43 (1981) 1253.

[74] P.S. Lejcek, S. Hofmann and V. Paidar, Acta Mater 51 (2003) 3951.

[75] R.L. Fullman, J. Appl. Phys. 22 (1951) 456.

[76]C.M. Sargent, Trans. AIME 242 (1968) 1188.

[77] R.C. Pond, J. de Physique. C4, 36 (1975) C4-315.

[78] D.M. Saylor, B.S. El-Dasher, T. Sano, and G.S. Rohrer, J. Amer. Ceram. Soc. in press. 
[79] D.M. Saylor, B.S. El-Dasher, Y. Pang, H.M. Miller, P. Wynblatt, A.D. Rollett, and G.S. Rohrer, J. Amer. Ceram. Soc. in press.

[80] D.M. Saylor, D.E. Mason and G.S. Rohrer, J. Amer. Ceram. Soc. 83 (2000) 1226.

[81] D.M. Saylor and G.S. Rohrer, Interface Science 9 (2001) 35.

[82] T. Sano, D.M. Saylor, and G.S. Rohrer, J. Amer. Ceram. Soc. 86 (2003) 1933.

[83]. J. Padilla and D. Vanderbilt, Surf. Sci. 418 (1998) 64.

[84]. A. Pojani, F. Finocchi, and C. Noguera, Surf. Sci. 442 (1999) 179.

[85]. H.A. Miers, Minerology, $2^{\text {nd }}$ Edition, Macmillan and Co., Ltd, London, (1929) p. $51,438$.

[86]. R.K. Mishra and G. Thomas, J. Appl. Phys. 48 (1977) 457.

[87]. C.M. Fang, S.C. Parker, and G. de With, J. Am. Ceram. Soc. 83 (2000) 2082.

[88]. H.A. Miers, Minerology, $2^{\text {nd }}$ Edition, Macmillan and Co., Ltd, London, (1929) p. 414.

[89]. M. Ramamoorthy, D. Vanderbilt, and R.D. King-Smith, Phys. Rev. B 49 (1994) 16721.

[90]. R.S. Nelson, D.J. Mazey, and R.S. Barnes, Philos. Mag. 11 (1965) 91.

[91] V. Randle, Mater. Charac. 47 (2001) 411.

[92] V. Randle, The Role of the Coincidence Site Lattice in Grain Boundary Engineering, Institute of Materials, London, (1996).

[93] G. Palumbo, E.M. Lehockey, and P. Lin, JOM Feb. (1998) 40.

[94] H. Davies and V. Randle, J. Microscopy 205 (2002) 253.

[95] S. Wright, J. Bingert, T.A. Mason, and R.J. Larsen, Mater. Sci. Forum 408-12 (2002) 511. 
[96] N.A. Gjostein and F.N. Rhines, Acta Metall. 7 (1959) 319.

[97] D.W. Readey and R.E. Jech, J. Amer. Ceram. Soc. 51 (1968) 201.

[98] G.C. Hasson and C. Goux, Scripta Metall. 5 (1971) 889.

[99] M. McLean, J. Mater. Sci. 8 (1973) 571.

[100] G. Dhalenne, A. Revcolevschi, and A Gervais, Phys. Stat. Sol (a) 56 (1979) 267.

[101] G. Dhalenne, M. Dechamps, and A. Revcolevschi, J. Amer. Ceram. Soc. 65 (1982)

C11.

[102] S. Kimura, E. Yasuda, and M. Sakaki, Yogyo-Kyokai-Shi 94 (1986) 795.

[103] D.M. Saylor, A. Morawiec, B.L. Adams, and G.S. Rohrer, Interface Science, 8 (2000) 131.

[104] J. Duyster and B. Stöckhert, Contrib. Mineral. Petrol. 140 (2001) 567.

[105] A. Otsuki, Acta Mater. 49 (2001) 1737.

[106] S.W. Chan and R.W. Balluffi, Acta Metall. 33 (1985) 1113.

[107] S.W. Chan and R.W. Balluffi, Acta Metall. 34 (1986) 2191.

[108] P. Chaudhari and J.W. Matthews, J. Appl. Phys. 42 (1971) 3063.

[109] D.M. Saylor, A. Morawiec, G.S. Rohrer, J. Amer. Ceram. Soc. 85 (2002) 3081.

[110] D.M. Saylor, A. Morawiec, and G.S. Rohrer, Acta Mater. 51 (2003) 3675.

[111] D. Wolf, J. Mater. Res. 5 (1990) 1708.

[112] A.P. Sutton, Prog. Mater. Sci. 36 (1992) 167.

[113] A.P. Sutton, Phil. Mag. A 63 (1991) 793.

[114] D Wolf and S. Phillpot, Mater. Sci. and Eng. A 107 (1989) 3. 
[115] W.T. Read and W. Shockley, Phys. Rev. 78 (1950) 275.

[116] F.C. Frank, in: A Symposium on the Plastic Deformation of Crystalline Solids,

Office of Naval Research, Washington, DC, (1950) 151.

[117] E.A. Holm, G.N. Hassold, and M.A. Miodownik, Acta Mater. 49 (2001) 2981.

[118] M. Upmanyu, G.N. Hassold, A Kazaryan, E.A. Holm, Y. Wang, B. Patton, D.J.

Srolovitz, Interface Science 10 (2002) 201.

\section{Correspondence address}

Gregory S. Rohrer

W.W. Mullins Professor of Materials Science and Engineering

Department of Materials Science and Engineering

Carnegie Institute of Technology

Carnegie Mellon University

5000 Forbes Ave.

Pittsburgh, Pennsylvania 15213-3890

phone: 412-268-2696

fax: 412-268-3113

e-mail: gr20@andrew.cmu.edu

www: http://neon.mems.cmu.edu/rohrer.html

\section{Figure Captions}

Figure 1. (a) Schematic shape a single grain from a dense polycrystal drawn after ref. [2].

(b) Representation of a three grain junction within a polycrystal. The view is exploded so that the internal interfaces can be seen. The external surfaces are shaded and the internal surfaces are triangulated. The $\mathrm{j}^{\text {th }}$ triangular facet on the $\mathrm{i}^{\text {th }}$ grain is shaded and an enlarged view of this facet is shown in (c).

Figure 2. The parameterization of $\square(\square g, \mathbf{n})$ into (a) two boundary plane orientation parameters and (b) three lattice misorientation parameters. (a) Definition of the spherical angles used to parameterize $\mathbf{n}$. The range of $\mathbf{n}$ is partitioned so that all of the cells have 
the same width in $\square$ and $\cos \square$ and the same area on the surface of the hemisphere. In the misorientaiton space, there are $\mathrm{D}^{3}$ cells and for each of these cells, there is a hemisphere of boundary plane normals with $4 \mathrm{D}^{2}$ cells.

Figure 3. A plot of $\square(\mathbf{n})$, the distribution of internal interfaces, in $\mathrm{MgO}$. The distribution is plotted in stereographic projection, and the (100), (110), and (111) poles are marked with a “+”, ““_", and triangle, respectively [4].

Figure 4. Approximate number of distinguishable grain boundaries as a function of the resolution $(\square)$ with which the parameters are measured.

Figure 5. The distribution of grain boundary planes in $\mathrm{MgO}$ for boundaries with a $45^{\circ}$ misorientation about [110], plotted in stereographic projection (a) along [001] and (b) along [110]. In (a), the misorientation axis is in the plane of the paper (at the position of the "“-") and in (b) it is perpendicular [4]. In (a) the zone of tilt boundaries is labeled with a t and the (100), (110), and (111) poles are marked with a "+", “-“, and triangle, respectively. In (b) the tilts are in the plane and the symmetric tilts are labeled st.

Figure 6. Discrete poles for $\square\left(\mathbf{n}_{1}, \mathbf{n}_{2}, \square\right)$. (a) range of orientations for $\mathbf{n}_{2}$ (b) range of orientations for $\mathbf{n}_{2}$. (c ) Grain boundary distribution in MRD for (111)\|(111) boundaries as a function of the twist angle [9]. Because of the discretization of $\square$, the population does not have the ideal symmetry for [111] misorientations.

Figure 7. The distribution of grain boundary planes in $\mathrm{MgO}$ for boundaries with a $45^{\circ}$ misorientation about [100], plotted in stereographic projection. The distribution of interface normals has $8 / \mathrm{m}$ point symmetry. The [100] rotation axis is in the plane of the paper and marked by the circle with "+".

Figure 8. Schematic illustration of a triple junction where three grain boundaries meet. 
Figure 9. A superposition of the grain boundary traces from adjacent layers in the microstructure of $\mathrm{MgO}$. The vertical separation is about $5 \square \mathrm{m}$ [4].

Figure 10. The distribution of grain boundary plane normals in $\mathrm{SrTiO}_{3}$ for boundaries with a misorientation $45^{\circ}$ about [100]. The stereogram in (a) is derived stereologically and the one in (b) is derived from serial sections. The populations, represented as multiples of a random distribution (MRD), are plotted in stereographic projection, with the [100], [110], and [111] directions marked with a '+', a '-', and a triangle, respectively [49].

Figure 11: The distribution of the differences (absolute value) between the recovered and actual grain boundary distributions from data sets consisting of $1 \times 10^{4}, 5 \times 10^{4}, 1 \times 10^{5}$, and $2.5 \times 10^{5}$ simulated boundaries [49].

Figure 12. Packing of tetrakaidecahedron.

Figure 13. The distribution of grain boundary planes averaged over all misorientations for (a) $\mathrm{SrTiO}_{3}$, (b) $\mathrm{MgAl}_{2} \mathrm{O}_{4}$, (c) $\mathrm{TiO}_{2}$, (d) Al. The data are shown in stereographic projection along [001], which is in the center of each projection. The [110] direction is marked with a square in each projection. In (c), the directions normal to the $\{101\}$ surfaces are marked with white diamonds and the directions normal to the $\{111\}$ surfaces are marked with white circles [79]

Figure 14. (a) Schematic sequence of tilt bicrystals with increasing misorientation about the [100] axis. If the black crystal maintains its interface plane on [001], then the interface plane of the gray crystal must tilt away from [001] by the misorientation angle. (b) The position of the normals of the black and gray crystal on a stereogram, where [001] is now points out of the plane and is in the center of the stereogram. (c) The 
populations for [100] tilt grain boundaries in $\mathrm{MgO}$, plotted on a [100] stereogram, at $5^{\circ}$ $15^{\circ}, 25^{\circ}$, and $35^{\circ}$. The zone of tilt boundaries is the vertical great circle in the center of each stereogram. At $35^{\circ}$, two separate grain boundary normal peaks are resolved [4].

Figure 15. Comparison of the reconstructed energies (squares) and the observed distribution (circles) for $\square 5$ tilt boundaries in $\mathrm{MgO}$. The quantities are plotted in $5^{\circ}$

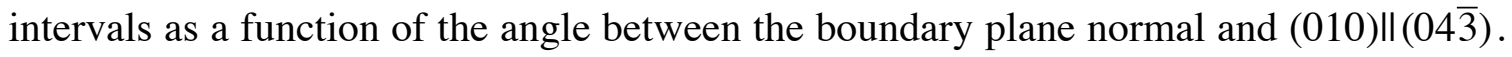
For reference, the location of the symmetric tilts, both the $\{310\}$ and $\{210\}$ types, as well as the asymmetric boundaries terminated by $\{100\}$ planes are indicated [110].

Figure 16. Observed grain boundary plane normal distributions for (a) $\square 3\left(60^{\circ} /[111]\right)$, (b) $\square 5\left(37^{\circ} /[100]\right)$, (c) $\square 7\left(38^{\circ} /[111]\right)$, and (d) $\square 9\left(39^{\circ} /[110]\right)$ misorientations. In each plot, the squares mark the position of the symmetric tilts and the circles the positions of the pure twist boundaries. (a) twist: (111), tilt: $(\overline{2} 11),(\overline{1} \overline{1} 2)$, and (1리) (b) twist: (100) and (100) tilt: (031), (012), (0 $\overline{1} 3)$, and $(0 \overline{2} 1)(c)$ twist: (111), tilt: $(\overline{3} 21),(\overline{2} \overline{1} 3)$ and $(1 \overline{3} 2)(d)$ twist $(110)$ and $(\overline{1} \overline{1} 0)$, tilt $(\overline{2} 21)$ and $(1 \overline{1} 4)$. The reference frame is the same as in Figs. 7, 10, and 14c [78].

Figure 17. Normalized values of the grain boundary population as a function of the reconstructed grain boundary energy. The average of all normalized values within a range of 0.032 a.u. is represented by the point; the bars indicate one standard deviation above and below the mean [109].

Figure 18. The average value of the $\square$ for $\mathrm{SrTiO}_{3}$ plotted as a function of the sum of the two surface energies. The circle represents the average population for all boundaries within a range of 0.012 a.u.; the bars indicate one standard deviation above and below the mean [78]. 
Figure 19. The observed distribution of grain boundary plane normals for boundaries with misorientations of (a) $20^{\circ}$, (b) $30^{\circ}$, and (c) $40^{\circ}$ around the [100] axis. The misorientation axis lies horizontally in the plane of the paper at the position of the [100] pole (indicated by the + ). For comparison, the hypothetical grain boundary energies based on the sum of the two surface energies (d-f) at the same fixed misorientations are also shown [78]. 


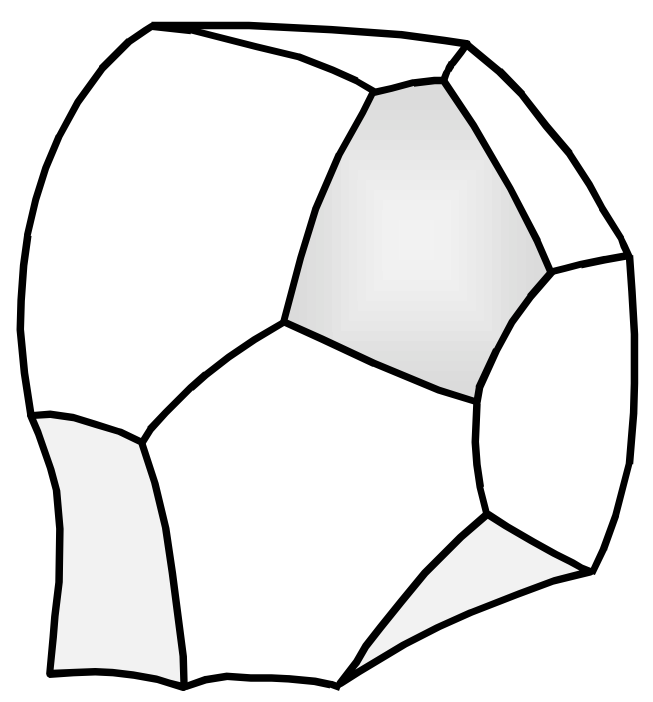

(a)

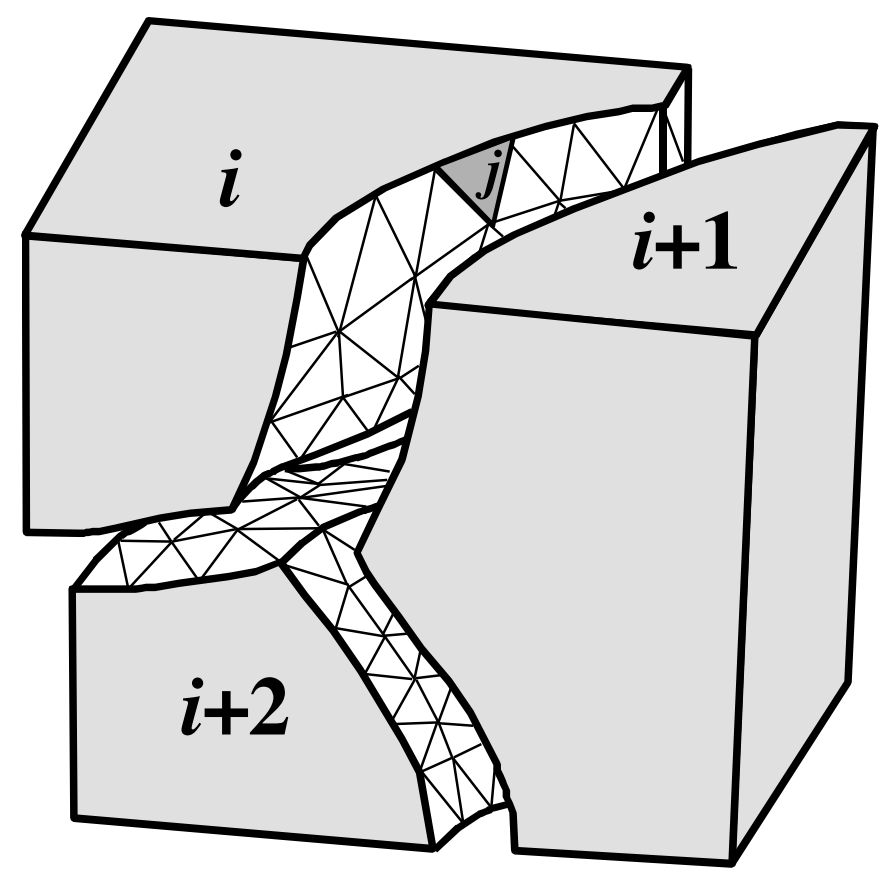

(b)

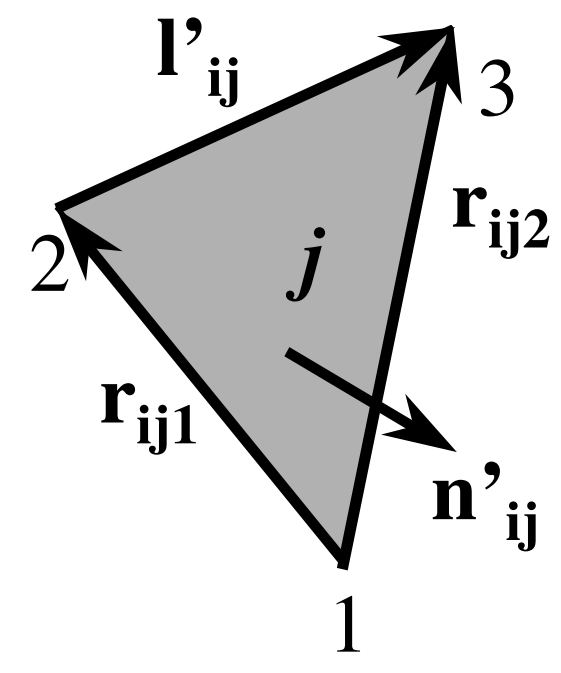

(c)

Figure 1. (a) Schematic shape a single grain from a dense polycrystal, drawn after ref. [2]. (b) Representation of a three grain junction within a polycrystal. The view is exploded so that the internal interfaces can be seen. The external surfaces are shaded and the internal surfaces are triangulated. The $\mathrm{j}^{\text {th }}$ triangular facet on the $\mathrm{i}^{\text {th }}$ grain is shaded and an enlarged view of this facet is shown in (c). 


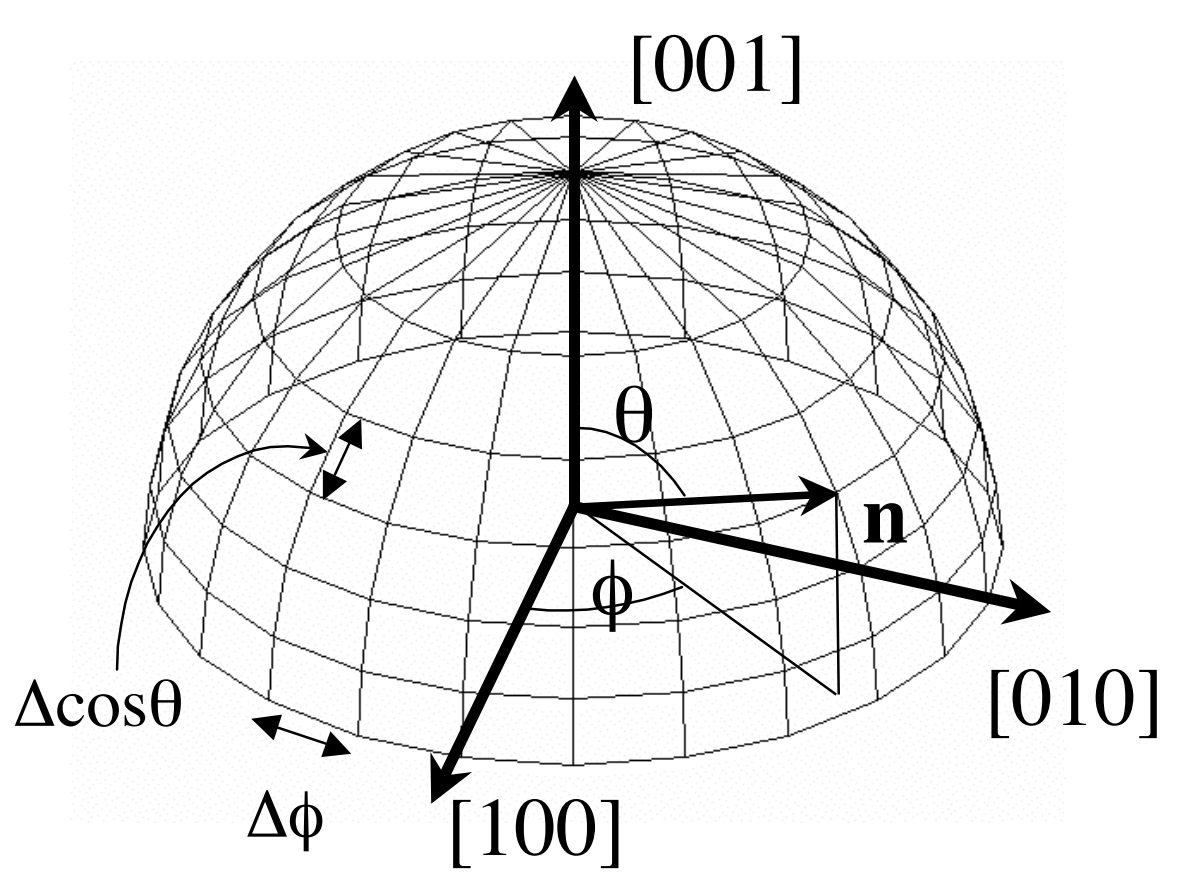

(a)

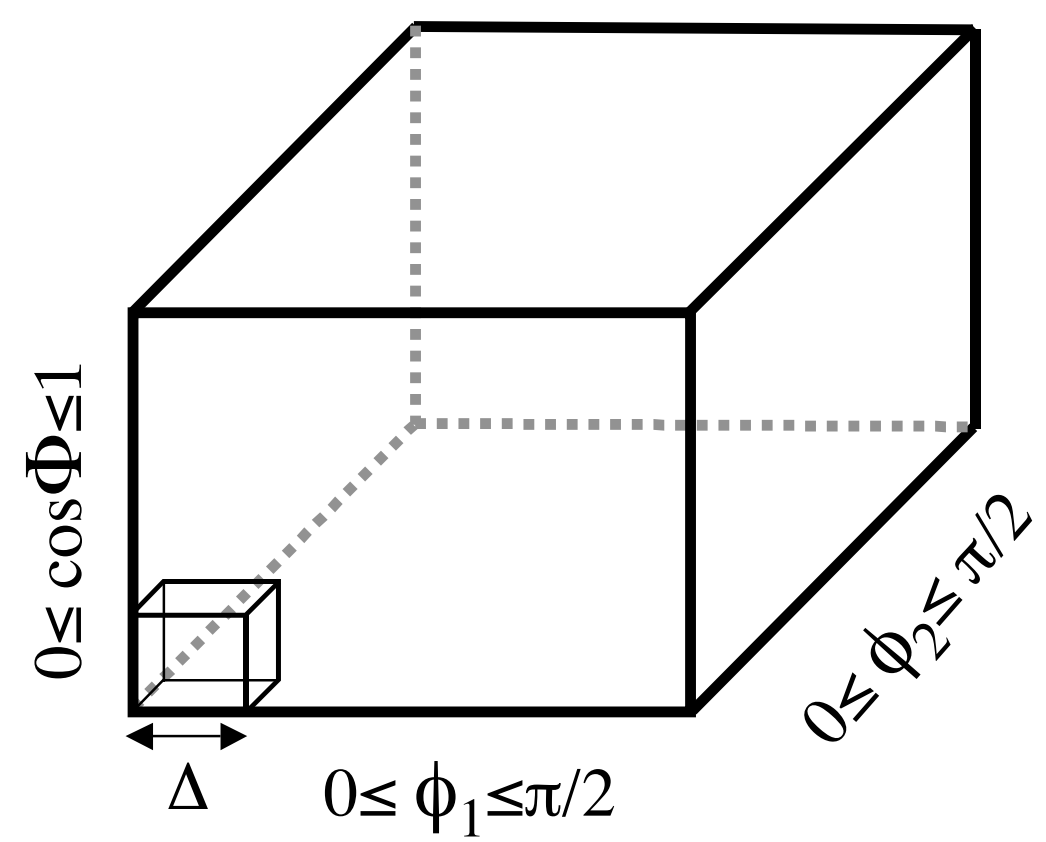

(b)

Figure 2. The parameterization of $\square(\mathrm{Dg}, \mathbf{n})$ into (a) two boundary plane orientation parameters and (b) three lattice misorientation parameters. (a) Definition of the spherical angles used to parameterize $\mathbf{n}$. The range of $\mathbf{n}$ is partitioned so that all of the cells have the same width in $\square$ and $\cos \square$ and the same area on the surface of the hemisphere. In the misorientaiton space, there are $\mathrm{D}^{3}$ cells and for each of these cells, there is a hemisphere of boundary plane normals with $4 \mathrm{D}^{2}$ cells. 


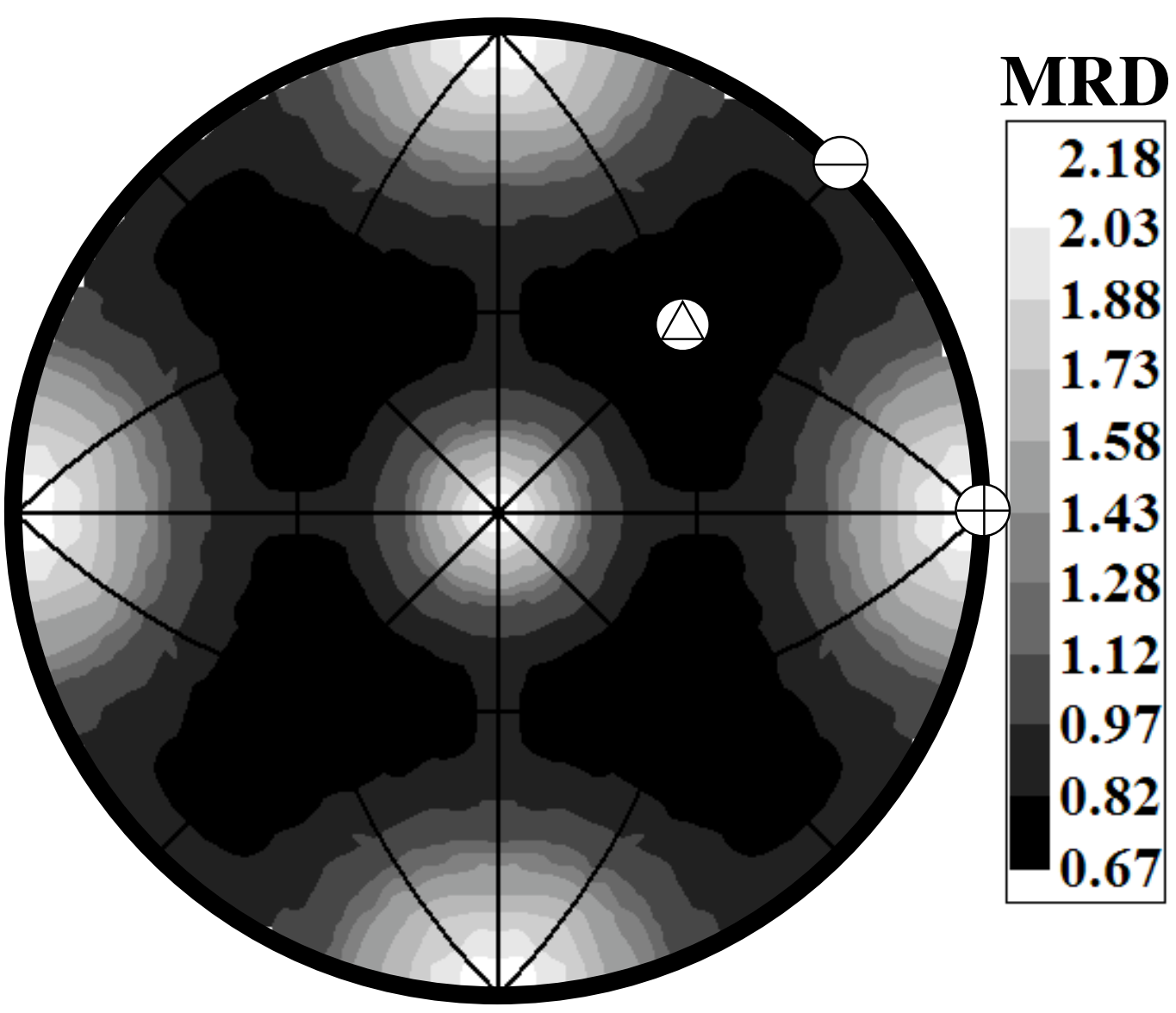

Figure 3. A plot of $\square(\mathbf{n})$, the distribution of internal interfaces, in $\mathrm{MgO}$. The distribution is plotted in stereographic projection, and the (100), (110), and (111) poles are marked with a “+”, “-“, and triangle, respectively [4]. 


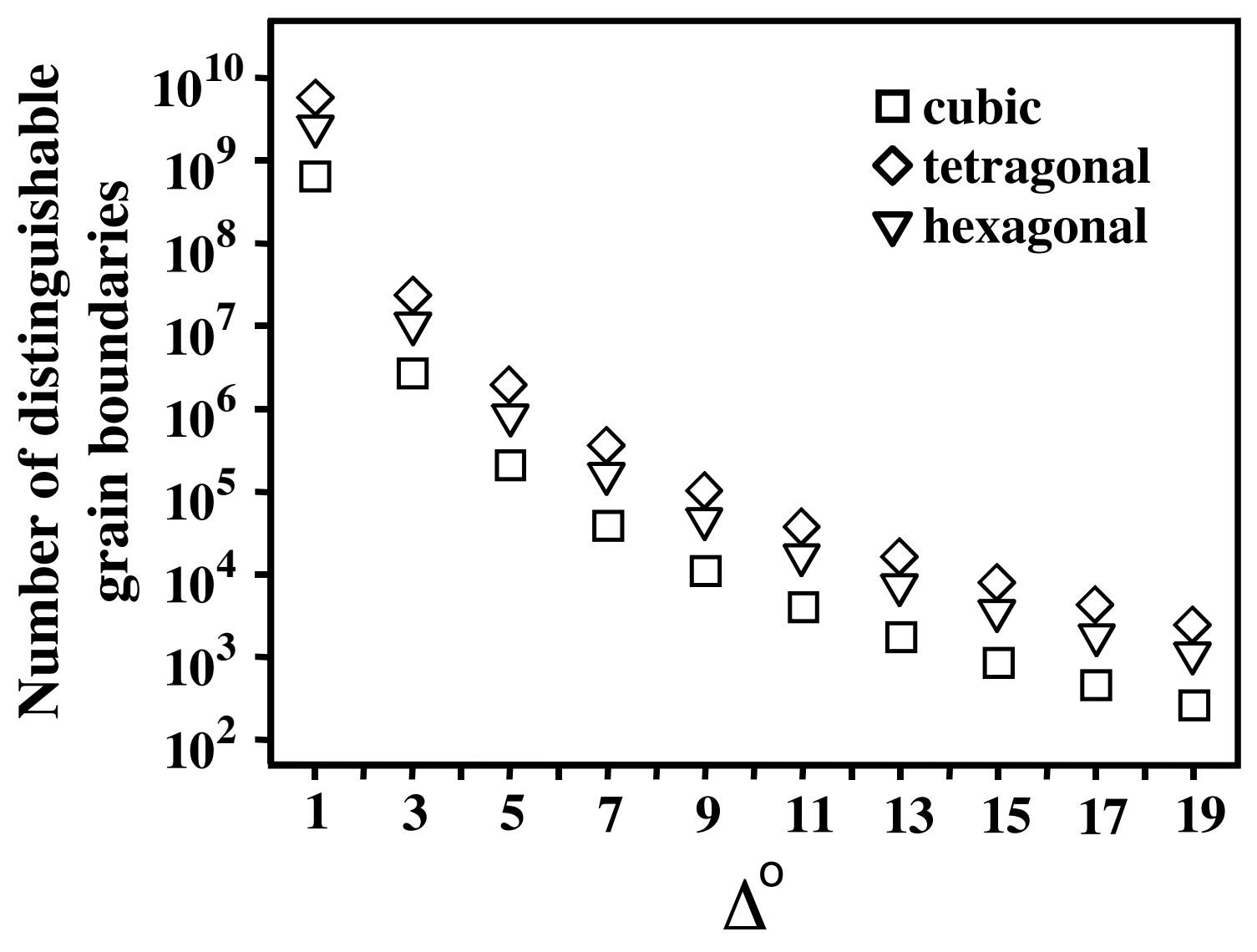

Figure 4. Approximate number of distinguishable grain boundaries as a function of the resolution ( $\square$ ) with which the parameters are measured. 


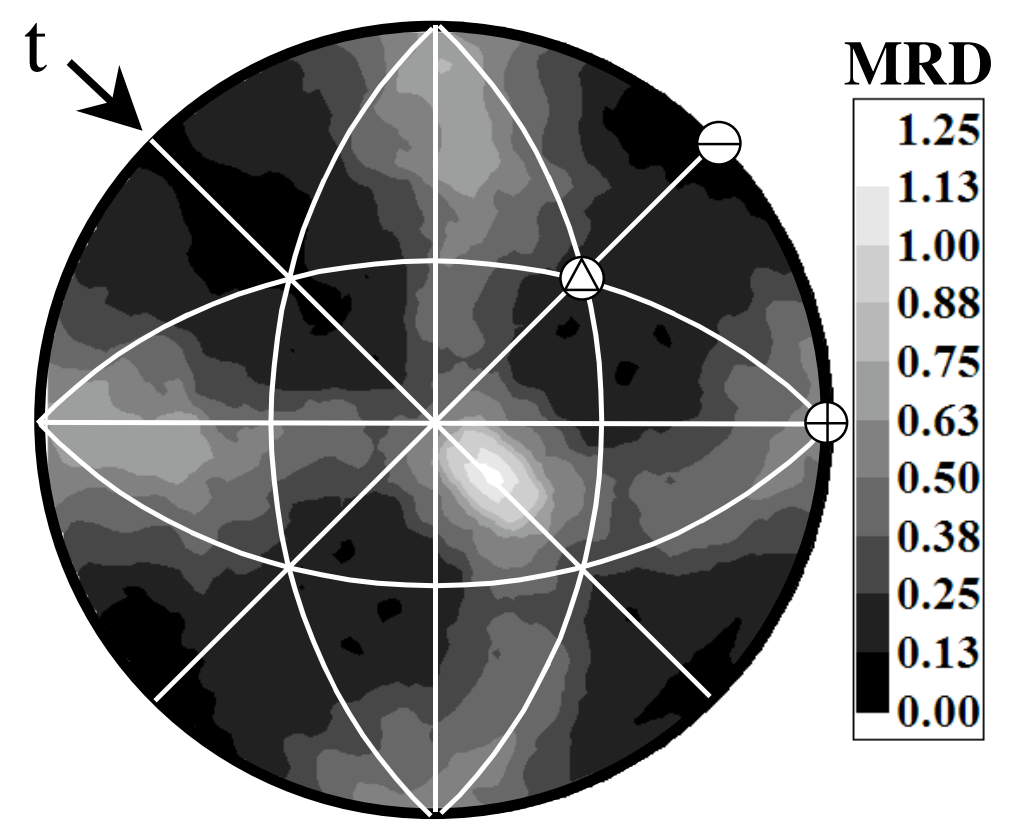

(a)

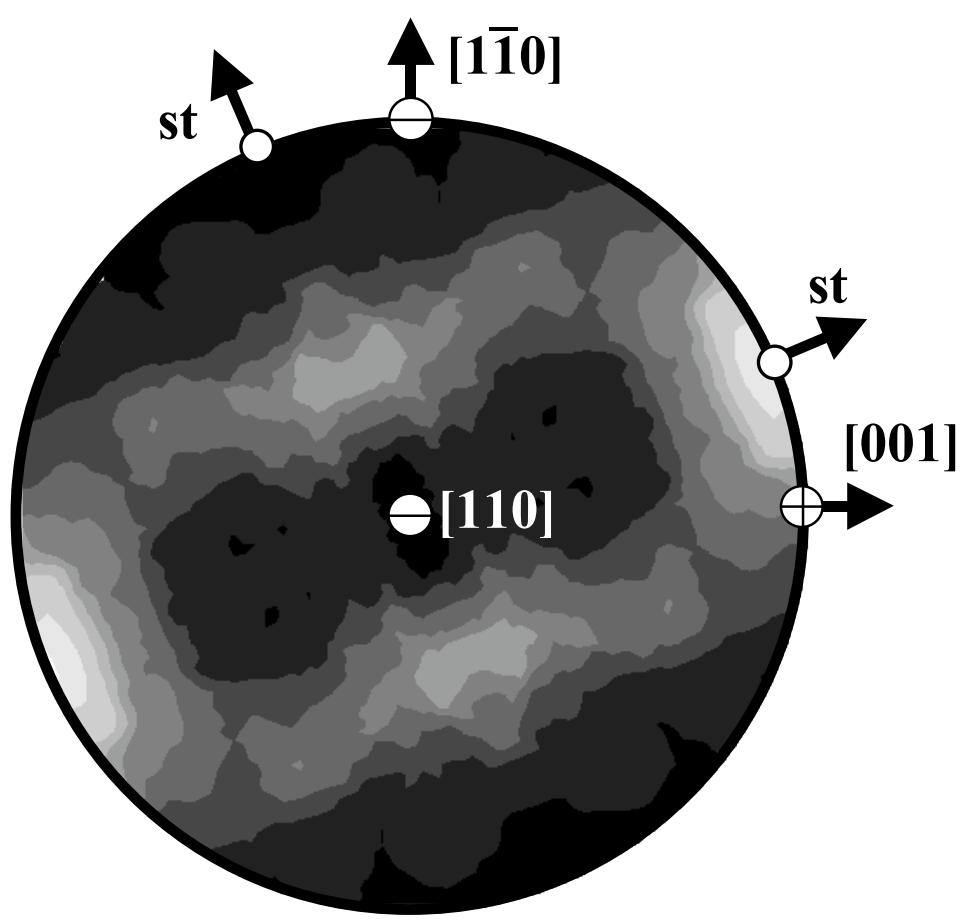

(b)

Figure 5. The distribution of grain boundary planes in $\mathrm{MgO}$ for boundaries with a $45^{\circ}$ misorientation about [110], plotted in stereographic projection (a) along [001] and (b) along [110]. In (a), the misorientation axis is in the plane of the paper (at the position of the "-“) and in (b) it is perpendicular [4]. In (a) the zone of tilt boundaries is labeled with a $\mathrm{t}$ and the (100), (110), and (111) poles are marked with a "+", “-“, and triangle, respectively. In (b) the tilts are in the plane and the symmetric tilts are labeled st. 


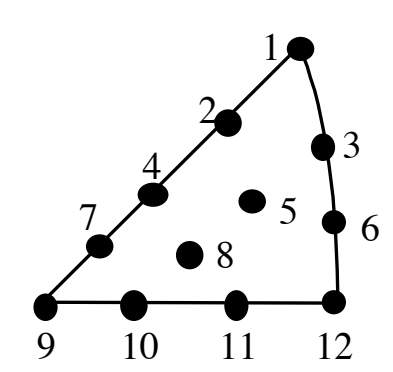

(a)

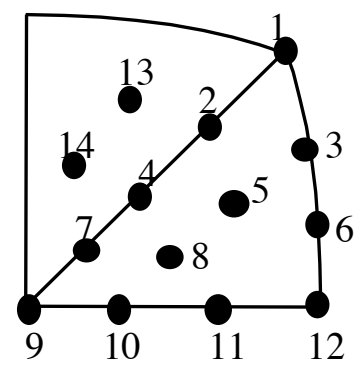

(b)
No. (hkl) No. (hkl)

$1:\left(\begin{array}{lll}1 & 1 & 1\end{array}\right) \quad 10:\left(\begin{array}{lll}15 & 4 & 0\end{array}\right)$

2:(5 33 ) 11:(5 30$)$

3:(7 74 4) 12:(1 110$)$

4:(3 111$)$ 13:(3 $\left.1 \begin{array}{lll}3 & 1\end{array}\right)$

5:(3 $\left.\begin{array}{lll}3 & 1\end{array}\right) \quad 14:\left(\begin{array}{lll}7 & 1 & 3\end{array}\right)$

6:(7 7 2)

7:(6 $\left.\begin{array}{lll}6 & 1 & 1\end{array}\right)$

8:(7 31 1)

9:( $\left.\begin{array}{lll}1 & 0 & 0\end{array}\right)$

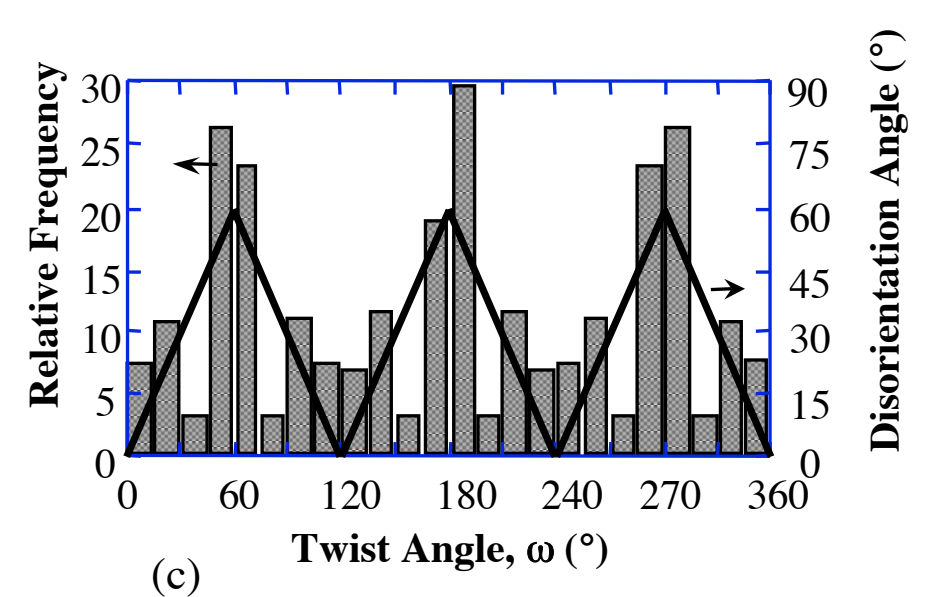

(c)

Figure 6. Discrete poles for $\square\left(\mathbf{n}_{1}, \mathbf{n}_{2}, \square\right)$. (a) range of orientations for $\mathbf{n}_{2}$ (b) range of orientations for $\mathbf{n}_{2}$. (c ) Grain boundary distribution in MRD for (111)\|(111) boundaries as a function of the twist angle [9]. Because of the discretization of $\square$, the population does not have the ideal symmetry for [111] misorientations. 


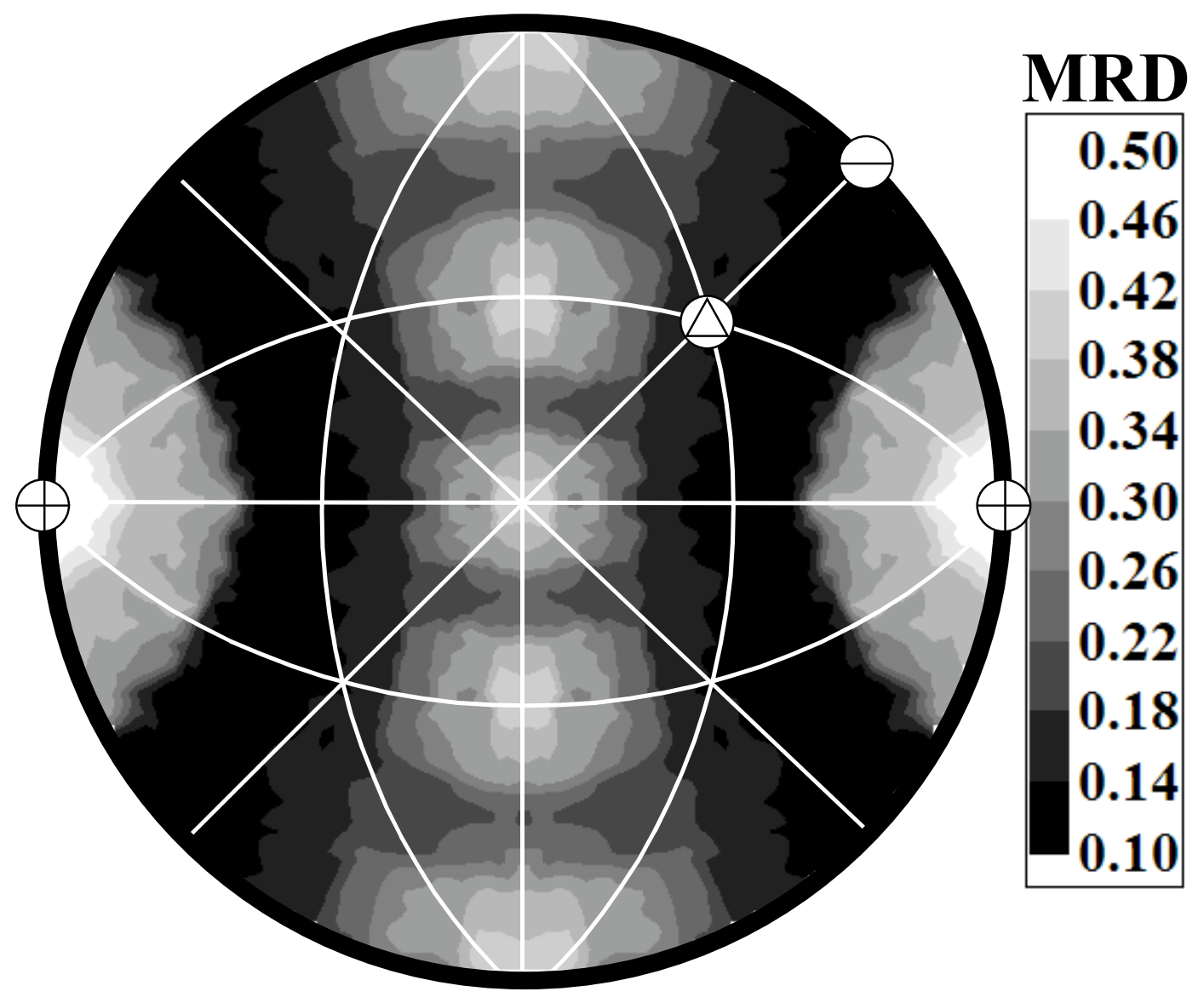

Figure 7. The distribution of grain boundary planes in $\mathrm{MgO}$ for boundaries with a $45^{\circ}$ misorientation about [100], plotted in stereographic projection. The distribution of interface normals has 8/m point symmetry. The [100] rotation axis is in the plane of the paper and marked by the circle with "+". 


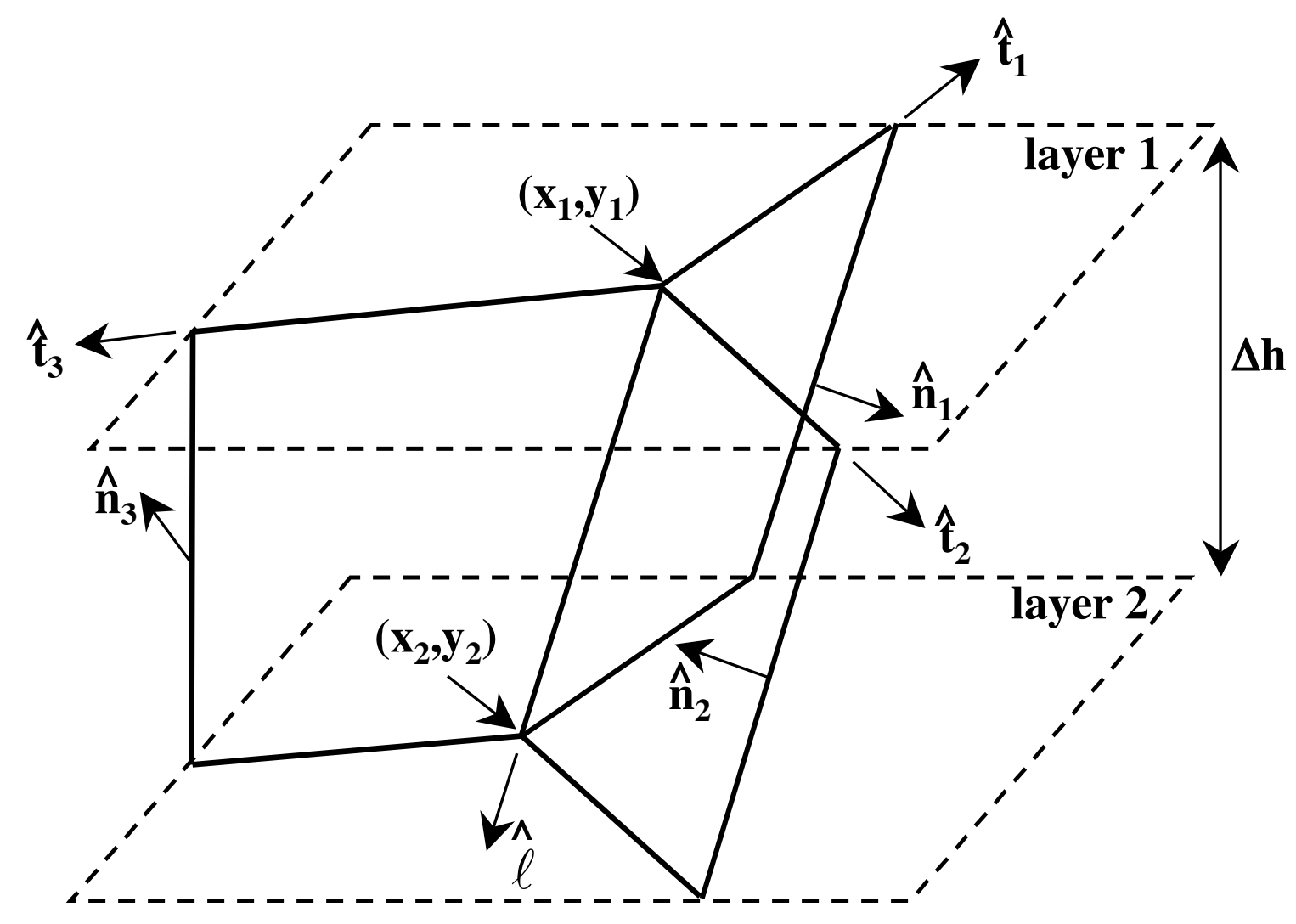

Figure 8. Schematic illustration of a triple junction where three boundary planes meet. 


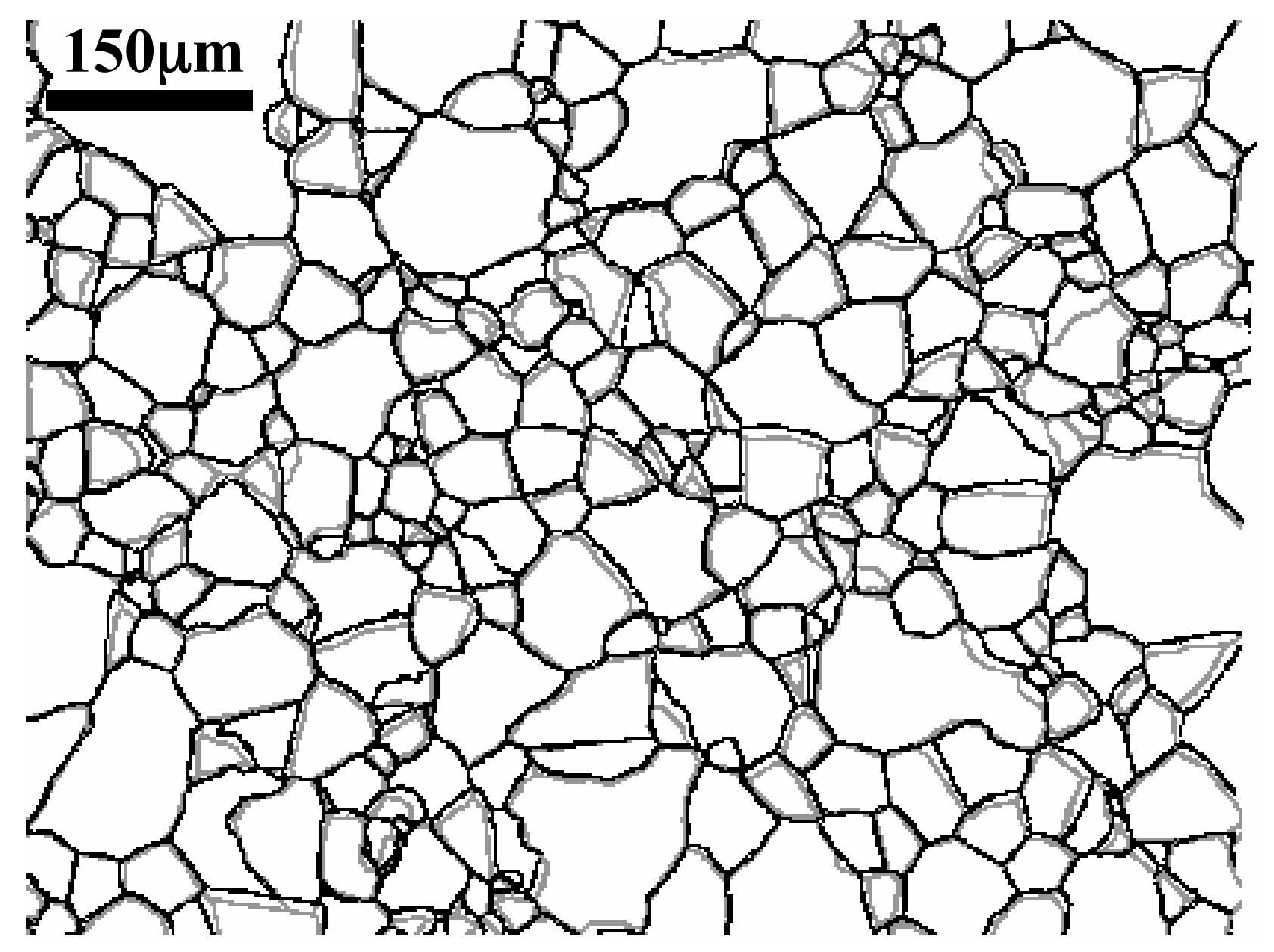

Figure 9. A superposition of the grain boundary traces from adjacent layers in the microstructure of $\mathrm{MgO}$. The vertical separation is about $5 \square \mathrm{m}$ [4]. 

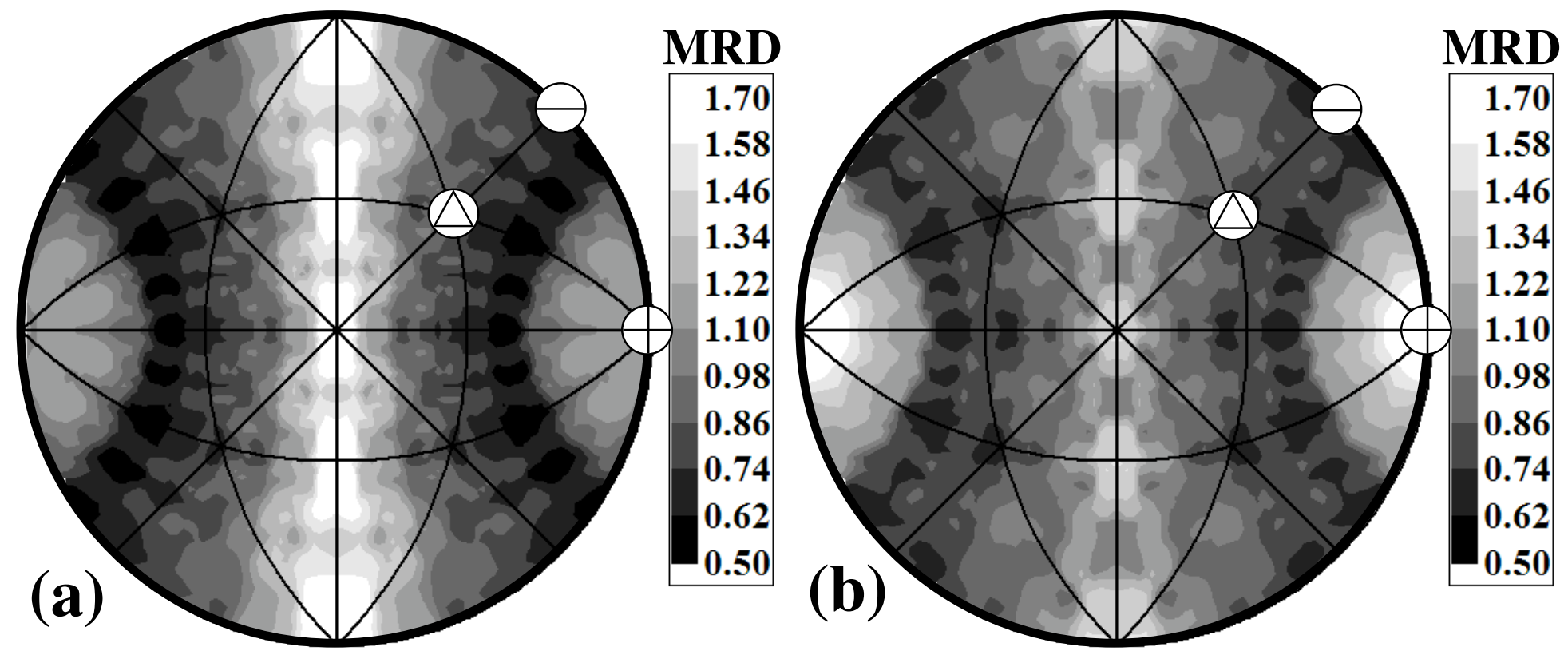

Figure 10. The distribution of grain boundary plane normals in $\mathrm{SrTiO}_{3}$ for boundaries with a misorientation $45^{\circ}$ about [100]. The stereogram in (a) is derived stereologically and the one in (b) is derived from serial sections. The populations, represented as multiples of a random distribution (MRD), are plotted in stereographic projection, with the [100], [110], and [111] directions marked with a '+', a '-', and a triangle, respectively [49]. 


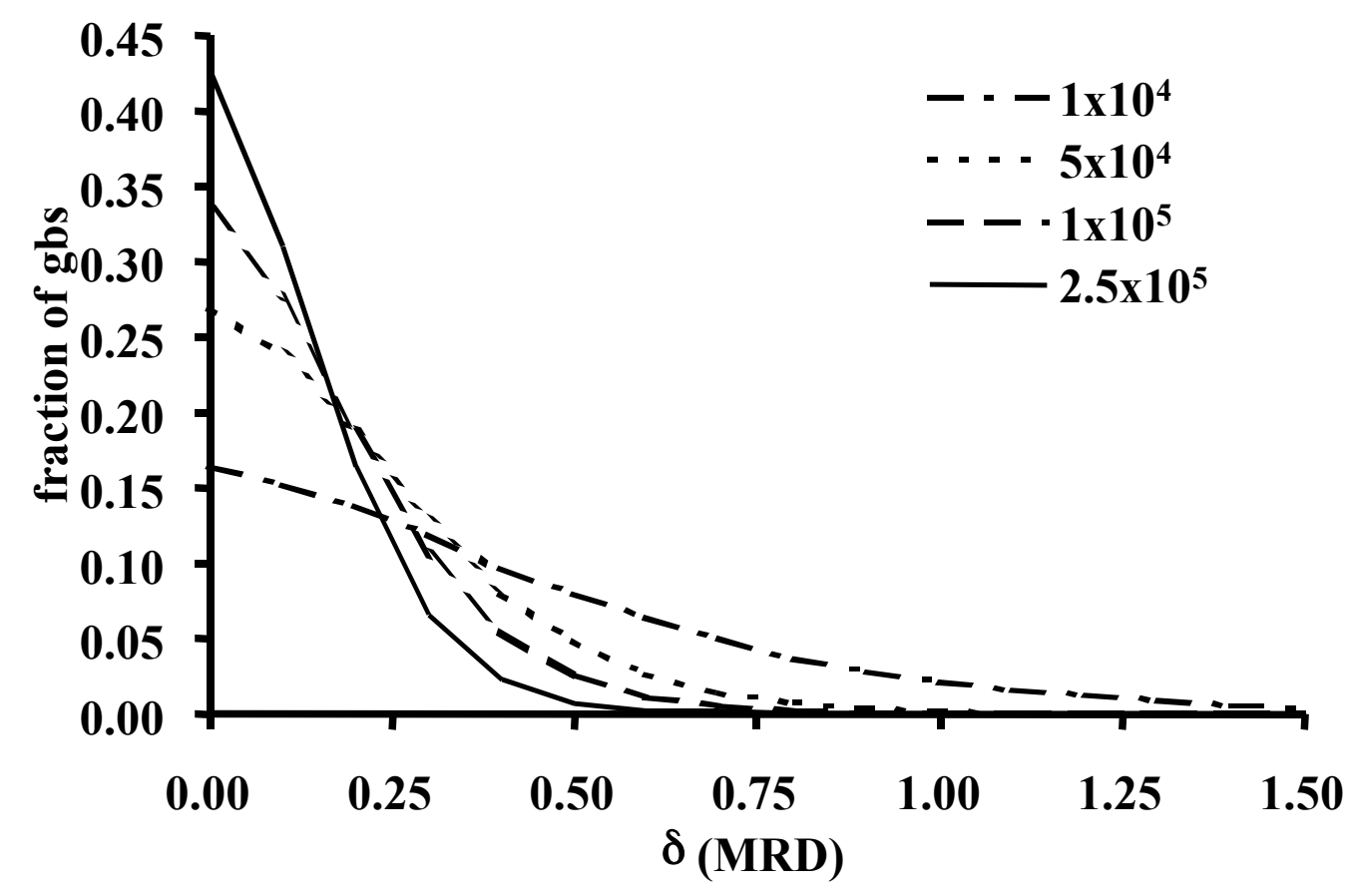

Figure 11: The distribution of the differences (absolute value) between the recovered and actual grain boundary distributions from data sets consisting of $1 \times 10^{4}, 5 \times 10^{4}, 1 \times 10^{5}$, and $2.5 \times 10^{5}$ simulated boundaries [49]. 


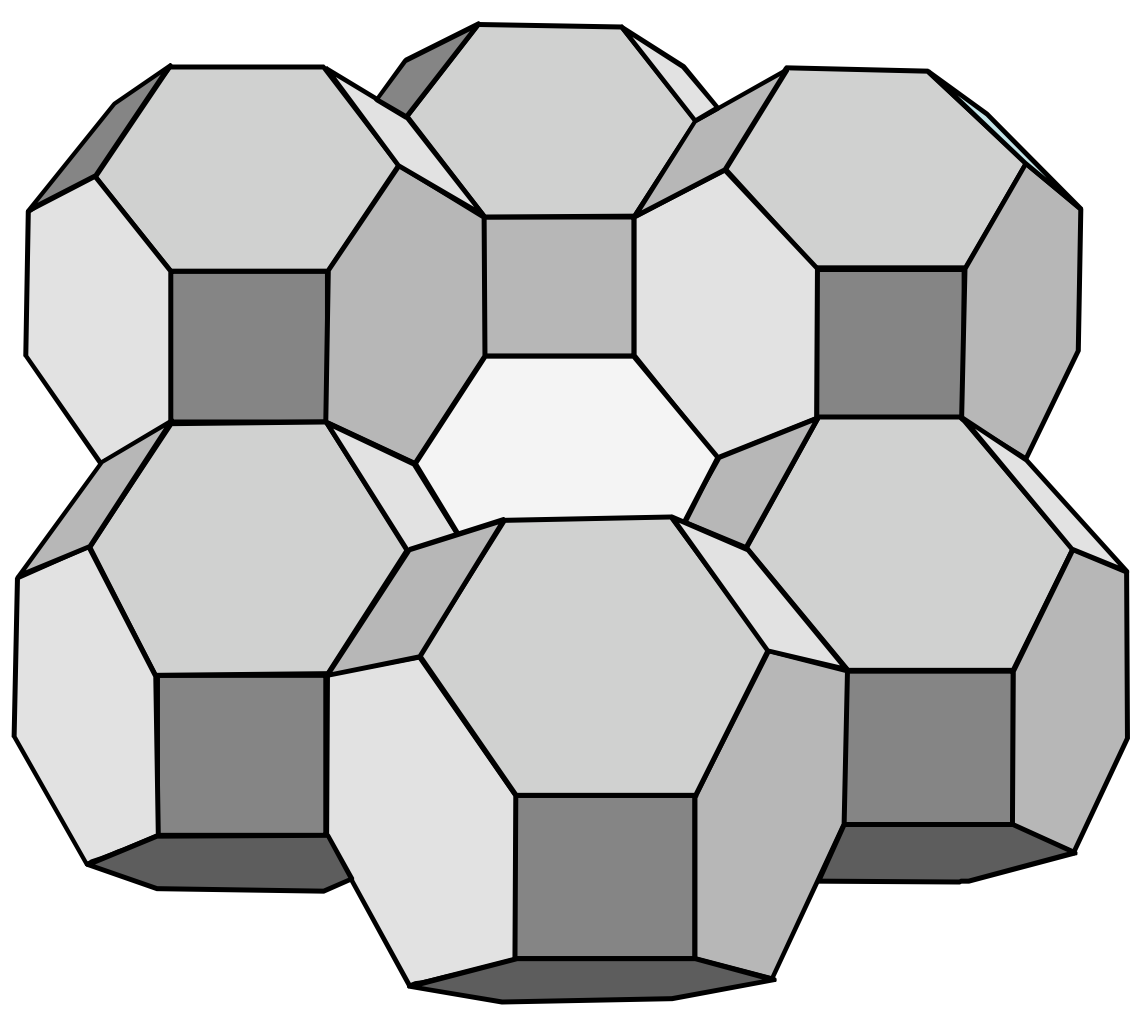

Figure 12. Packing of tetrakaidecahedron. 

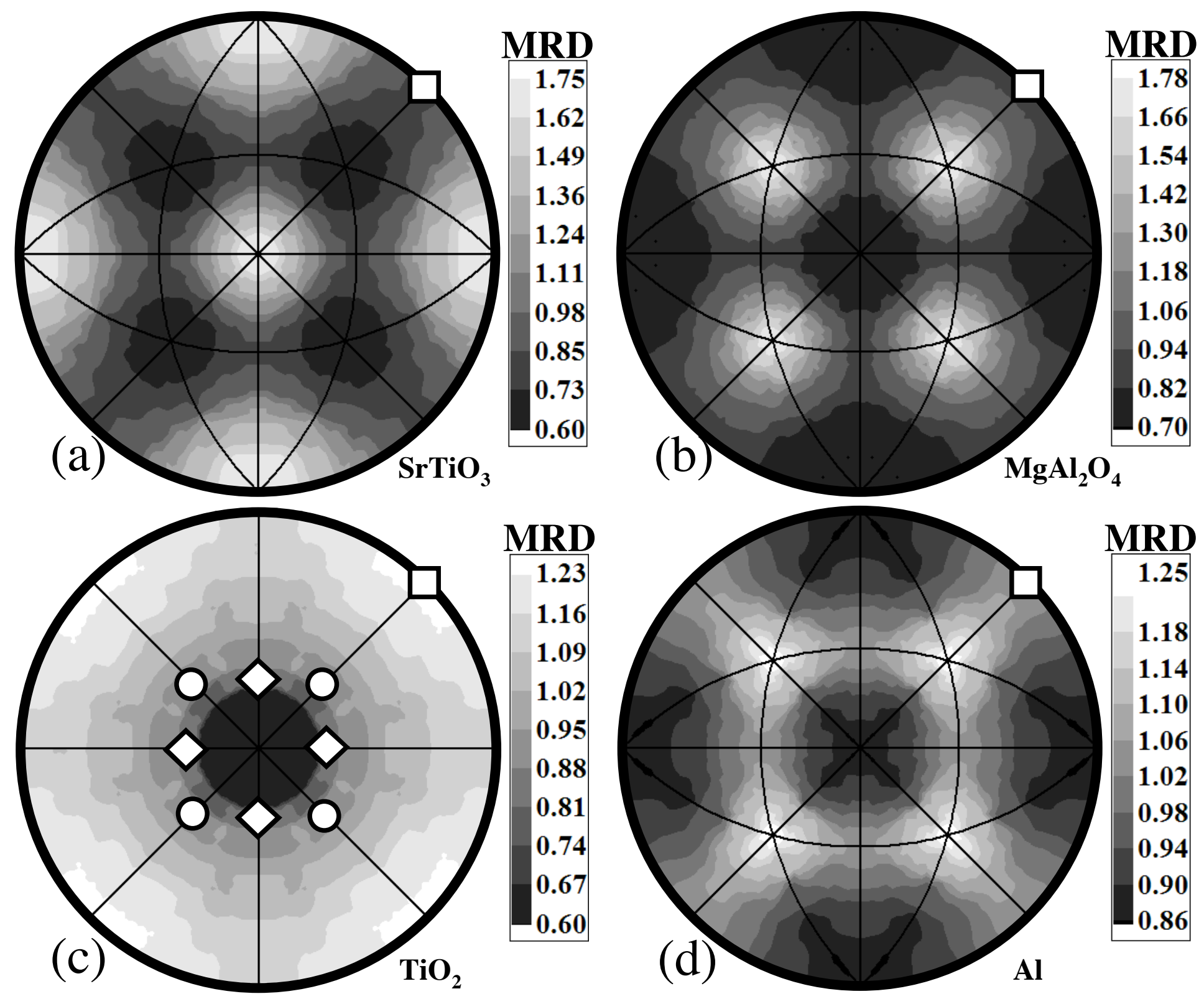

Figure 13. The distribution of grain boundary planes averaged over all misorientations for (a) $\mathrm{SrTiO}_{3},(\mathbf{b}) \mathrm{MgAl}_{2} \mathrm{O}_{4},(\mathbf{c}) \mathrm{TiO}_{2},(\mathbf{d})$ Al. The data are shown in stereographic projection along [001], which is in the center of each projection. The [110] direction is marked with a square in each projection. In (c), the directions normal to the $\{101\}$ surfaces are marked with white diamonds and the directions normal to the $\{111\}$ surfaces are marked with white circles [79]. 
(a)
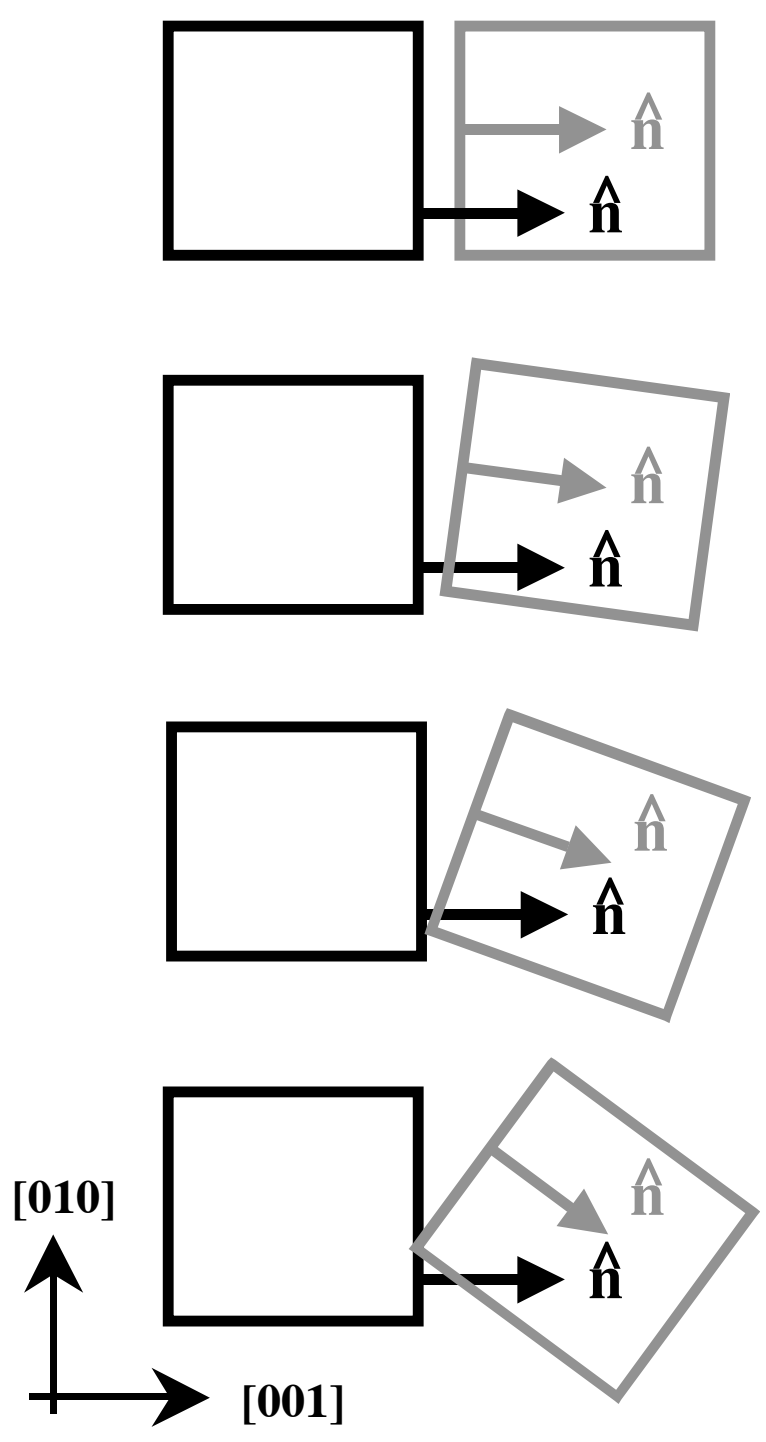

(b)
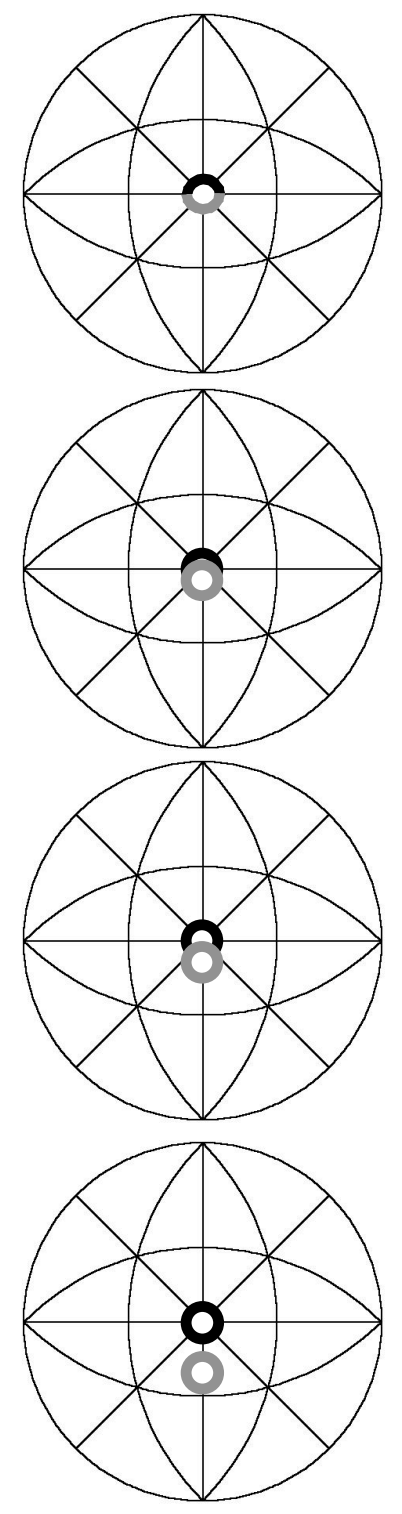

(c)
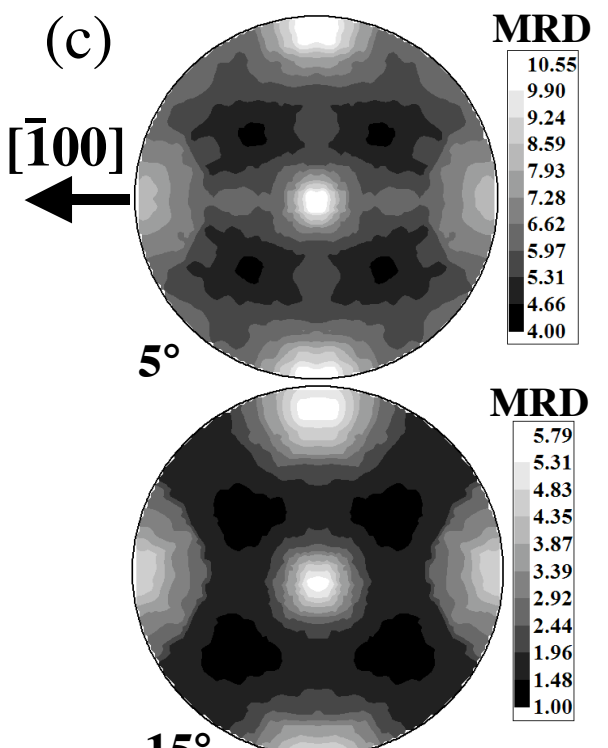

$15^{\circ}$

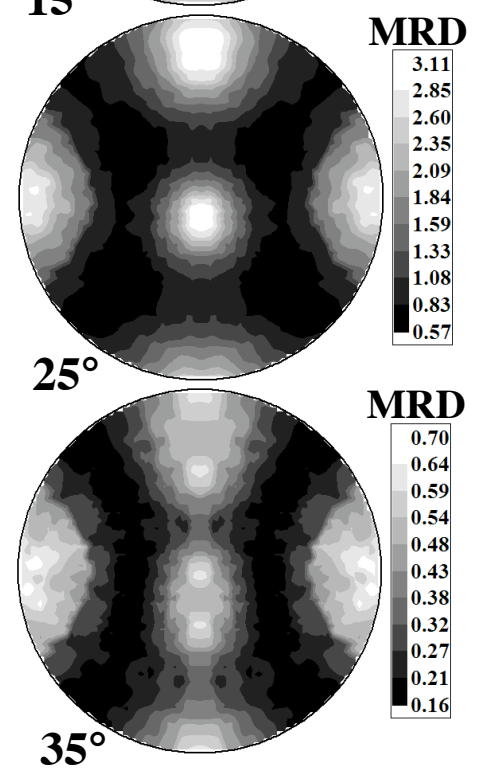

Figure 14. (a) Schematic sequence of tilt bicrystals with increasing misorientation about the [100] axis. If the black crystal maintains its interface plane on [001], then the interface plane of the gray crystal must tilt away from [001] by the misorientation angle. (b) The position of the normals of the black and gray crystal on a stereogram, where [001] is now points out of the plane and is in the center of the stereogram.

(c) The populations for [100] tilt grain boundaries in $\mathrm{MgO}$, plotted on a [100] stereogram, at $5^{\circ} 15^{\circ}, 25^{\circ}$, and $35^{\circ}$. The zone of tilt boundaries is the vertical great circle in the center of each stereogram. At $35^{\circ}$, two separate grain boundary normal peaks are resolved [4]. 


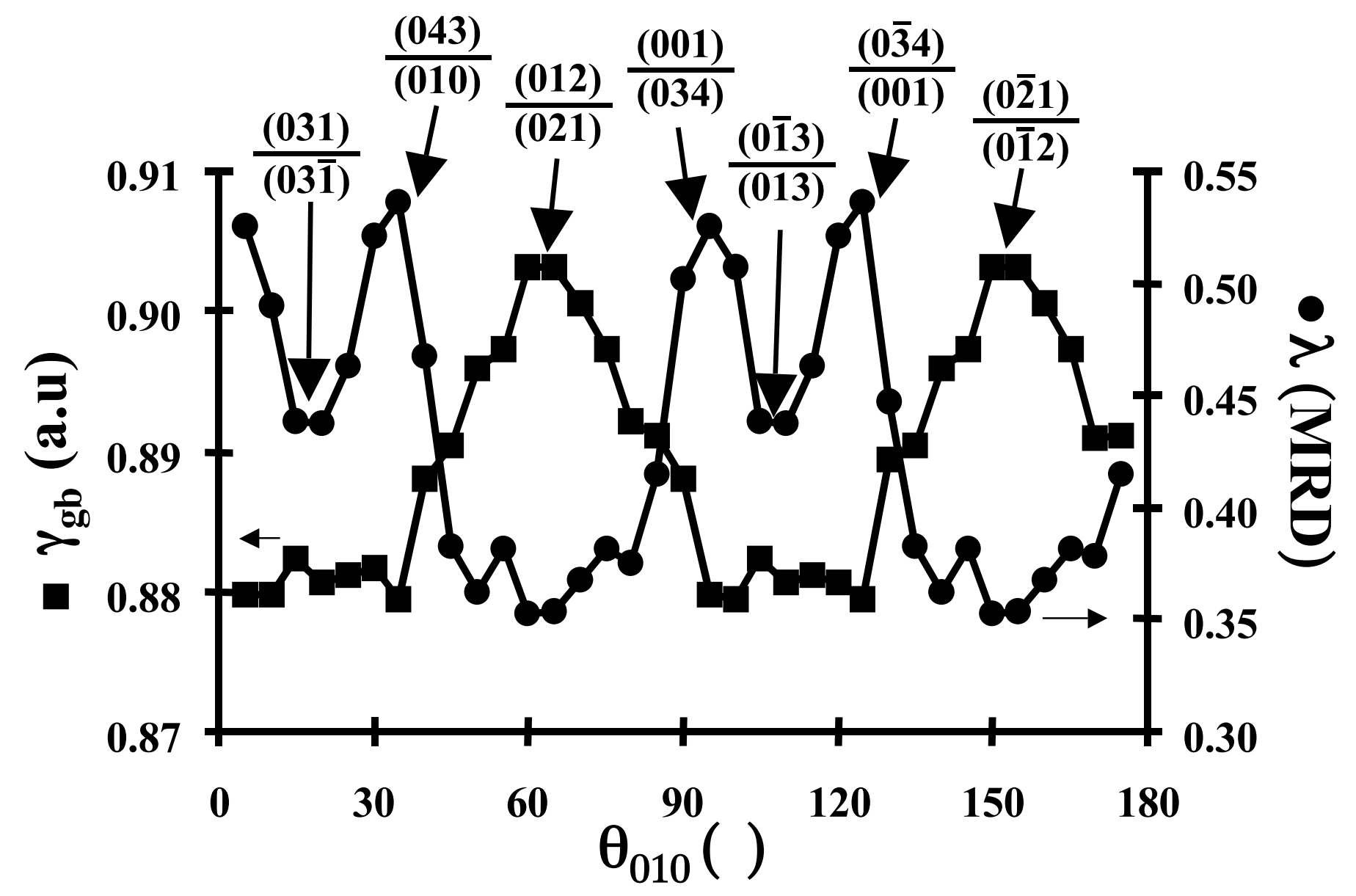

Figure 15. Comparison of the reconstructed energies (squares) and the observed distribution (circles) for $\square 5$ tilt boundaries in $\mathrm{MgO}$. The quantities are plotted in $5^{\circ}$ intervals as a function of the angle between the boundary plane normal and $(010) \|(04 \overline{3})$. For reference, the location of the symmetric tilts, both the $\{310\}$ and $\{210\}$ types, as well as the asymmetric boundaries terminated by $\{100\}$ planes are indicated [110]. 

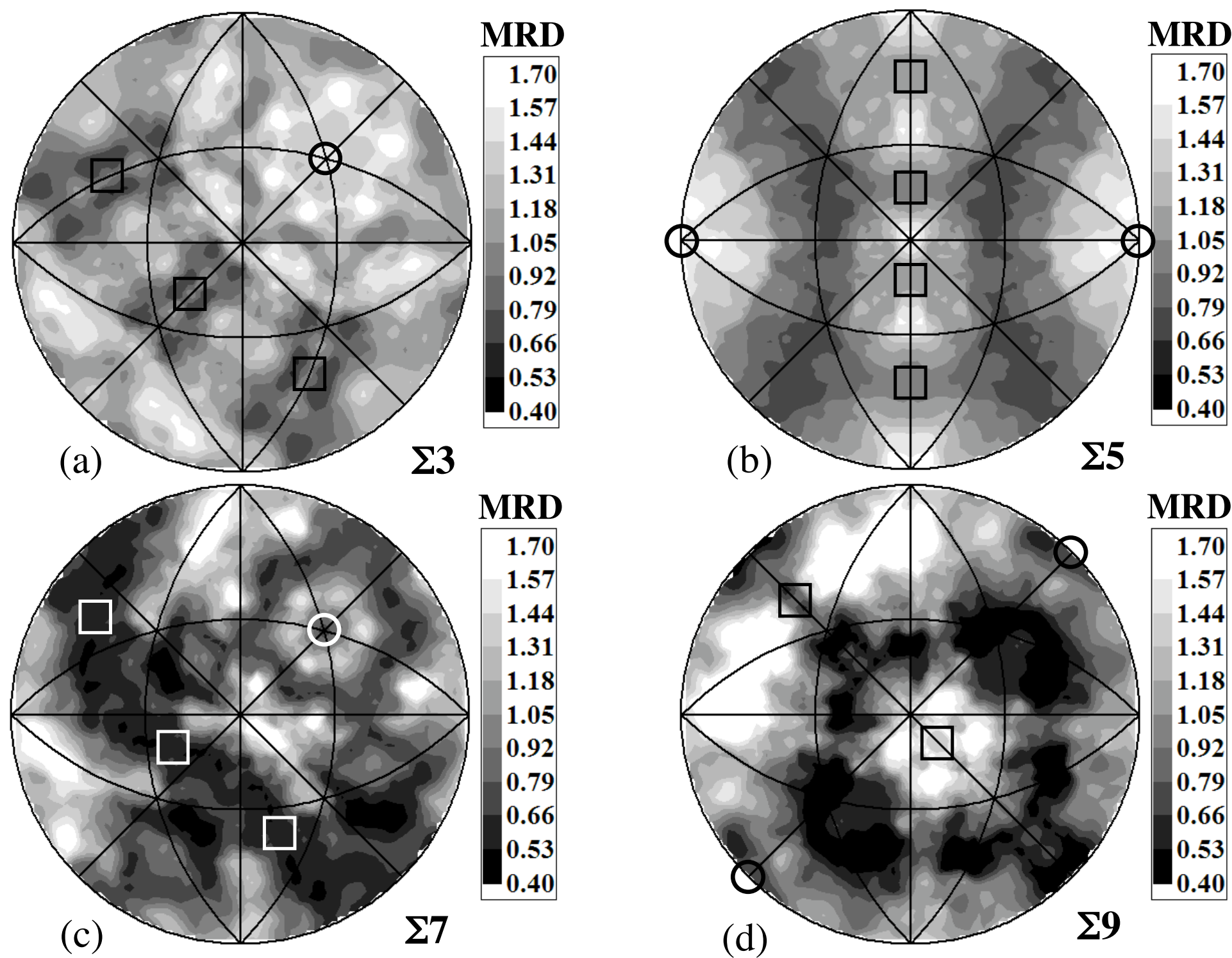

Figure 16. Observed grain boundary plane normal distributions for (a) $\square 3\left(60^{\circ} /[111]\right)$, (b) $\square 5$ (37\%/[100]), (c) $\square 7$ (38\%/[111]), and (d) $\square 9\left(39^{\circ} /[110]\right)$ misorientations. In each plot, the squares mark the position of the symmetric tilts and the circles the positions of the pure twist boundaries. (a) twist: (111), tilt: $(\overline{2} 11),(\overline{1} \overline{1} 2)$, and (1 $\overline{2} 1)$ (b) twist: (100) and ( $\overline{1} 00)$ tilt: (031), (012), $(0 \overline{1} 3)$, and $(0 \overline{2} 1)$ (c) twist: (111), tilt: $(\overline{3} 21),(\overline{2} \overline{1} 3)$ and $(1 \overline{3} 2)(\mathrm{d})$ twist (110) and ( $\overline{1} \overline{1} 0)$, tilt $(\overline{2} 21)$ and $(1 \overline{1} 4)$. The reference frame is the same as in Figs. 7, 10, and 14c [78]. 


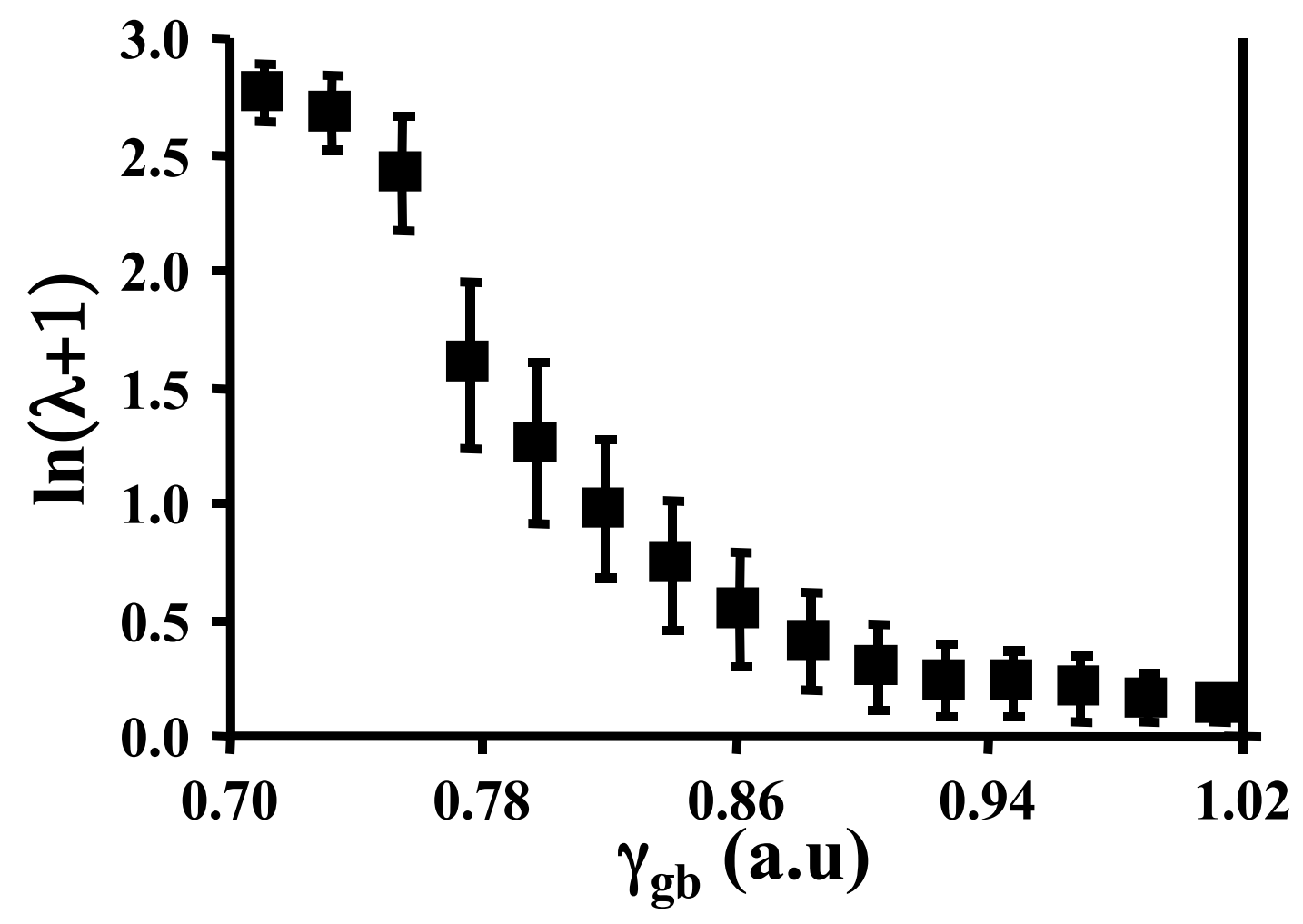

Figure 17. Normalized values of the grain boundary population as a function of the reconstructed grain boundary energy. The average of all normalized values within a range of 0.032 a.u. is represented by the point; the bars indicate one standard deviation above and below the mean [109]. 


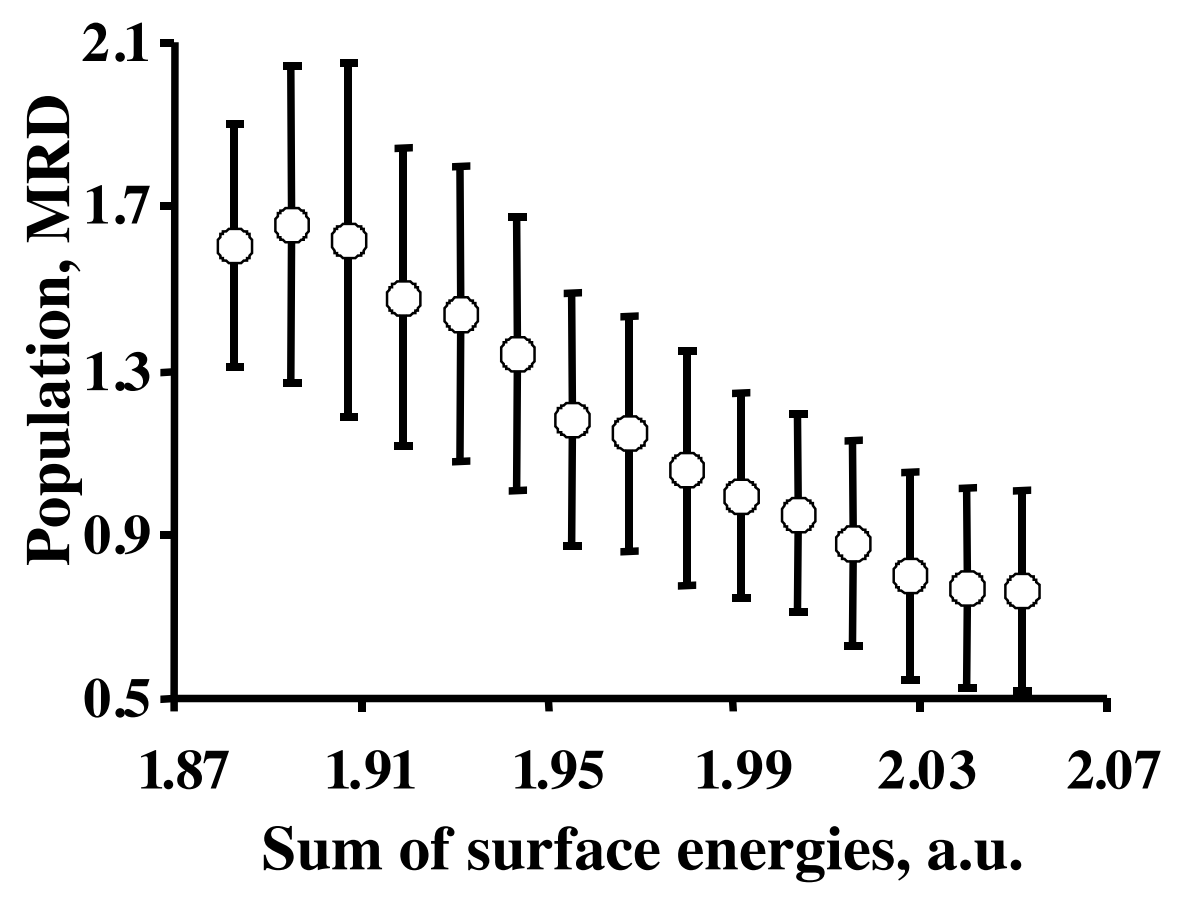

Figure 18. The average value of the $\square$ for $\mathrm{SrTiO}_{3}$ plotted as a function of the sum of the two surface energies. The circle represents the average population for all boundaries within a range of 0.012 a.u.; the bars indicate one standard deviation above and below the mean [78]. 

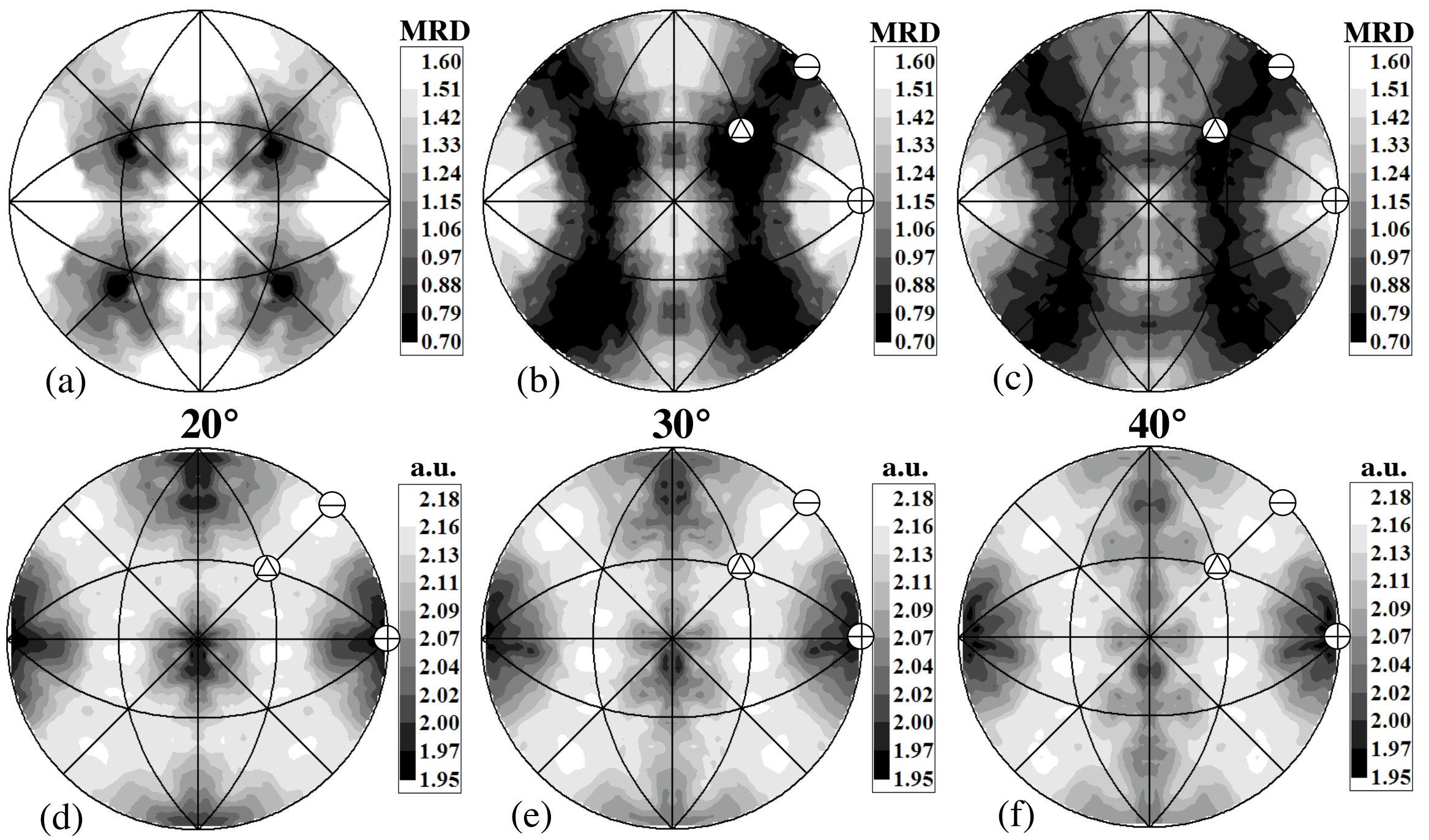

Figure 19. The observed distribution of grain boundary plane normals for boundaries with misorientations of (a) $20^{\circ}$, (b) $30^{\circ}$, and (c) $40^{\circ}$ around the [100] axis. The misorientation axis lies horizontally in the plane of the paper at the position of the [100] pole (indicated by the +). For comparison, the hypothetical grain boundary energies based on the sum of the two surface energies (d-f) at the same fixed misorientations are also shown [78]. 